artículos derivados de proyectos de investigación 

CUAD. CONTAB. / BOGOTÁ, COLOMBIA, 17 (44): 317-348 / JULIO-DICIEMBRE 2016 / 317

\title{
Industria automotriz de Colombia: ¿un motor generador de valor económico agregado?*
}

doi:10.11144/Javeriana.cc17-44.iacm

\author{
Ana Milena Padilla-Ospina \\ Estudiante de doctorado en administración. Magíster \\ en ciencias de la organización. Investigadora del grupo \\ de investigación en Generación de Valor Económico, \\ Universidad del Valle. \\ Correo electrónico: ana.milena.padilla@correounivalle. \\ edu.co
}

\section{Jorge Alberto Rivera-Godoy}

Profesor titular, Universidad del Valle. Doctor distinguido cum laude en ciencias económicas y empresariales, Universidad Autónoma de Madrid, UAM. Especialista en finanzas. Coordinador del grupo de investigación en Generación de Valor Económico, Universidad del Valle. Correo electrónico: jorge.rivera@correounivalle.edu.co

\footnotetext{
* Artículo resultado del proyecto de investigación Evaluación del desempeño financiero del sector automotriz en Colombia, realizado entre julio de 2014 y julio de 2015 por el grupo de investigación en Generación de Valor Económico, Universidad del Valle.
} 


\section{Resumen Este artículo tiene como propósito conocer} el desempeño financiero del sector automotor de Colombia durante el período 2008-2014 mediante un estudio de su eficiencia, eficacia, efectividad y valor económico agregado, siguiendo como metodología el análisis de los indicadores financieros con base en información contable y los relacionados con la gestión del valor económico. Este sector genera rentabilidades contables en cada año analizado, $y$ el comportamiento de su efectividad, en orden de importancia, depende de la eficacia, el apalancamiento financiero y la eficiencia; no obstante, tan solo en un año crea valor económico agregado (EVA) y su valor de mercado agregado (VMA) es negativo. El comportamiento del EVA está más relacionado con la orientación del indicador de rentabilidad del activo neto operacional que con la del costo de capital medio ponderado; y el primero depende de la eficacia en el control de costos y gastos, y de la eficiencia en el uso de los activos netos operacionales. A pesar de que en esta investigación se muestran los factores que han influido en el desempeño del sector automotor colombiano, estos resultados podrían fortalecerse si se pudieran hacer estudios similares para grupos de empresas más homogéneos por edad, tamaño y ubicación geográfica.

Palabras clave Valor económico agregado (EVA); valor de mercado agregado (VMA); desempeño financiero; análisis financiero; indicadores financieros de eficiencia, efectividad y eficacia; inductores del EVA; industria automotriz

Códigos JEL G10, M40, L62

\section{Automotive Industry in Colombia: an Engine that Generates Added Economic Value?}

\footnotetext{
Abstract The purpose of this article is to assess the financial performance of the Colombian automotive sector during the 2008-2014 period by studying its efficiency, efficacy, effectiveness, and economic value added, using a methodology relying on the analysis of financial indicators
}

based on accounting data, and those related to the management of economic value added. This sector generates accounting returns in each of the years analyzed, and the performance of its effectiveness -in order of importancedepends on efficacy, financial leverage, and efficiency. Still, in only one year it creates economic value added (EVA) and its market value added (MVA) is negative. The performance of the EVA has a closer relationship to the orientation of the profitability indicator of net operating assets than to the weighted average cost of capital. The first depends on the efficacy of the control exerted over costs and expenses, and the efficiency of the use of net operating assets. Although this study shows the factors that have influenced the performance of the Colombian automotive industry, these results could be reinforced if similar studies could be done for more homogeneous groups of companies classified by age, size, and geographical location.

Keywords Economic Value Added (EVA); Market Value Added (MVA); financial performance; financial analysis; financial indicators of efficiency, effectiveness, and efficacy; EVA induces; automotive industry

\section{Indústria automotiva da Colômbia: motor gerador de valor económico adicionado?}

Resumo Este artigo objetiva conhecer o desempenho financeiro do setor automotor da Colômbia durante o período 2008-2014 mediante estudo da eficiência, eficácia, efetividade e valor económico adicionado, seguindo a metodologia de análise dos indicadores financeiros baseados em informações contábeis e os relacionados com gestão de valor económico. Este setor gera retornos contábeis para cada ano analisado e o comportamento da sua efetividade, em ordem de importância, depende da eficácia, a alavancagem financeira e a eficiência; no entanto, apenas em um ano cria valor económico adicionado (EVA) e o valor de mercado agregado (VMA) é negativo. O comportamento do EVA está mais relacionado com a orientação do indicador de rentabilidade do ativo neto operacional quanto com a do custo de capital médio ponde- 
rado; e o primeiro depende da eficácia no controlo de custos e gastos e da eficiência no uso dos ativos netos operacionais. Embora nesta pesquisa sejam mostrados os fatores que influíram no desempenho do setor automóvel colombiano, estes resultados poderiam se fortalecer caso estudos similares pudessem ser feitos para grupos de empresas mais homogéneos por idade, comprimento e localização geográfica.

Palavras-chave Valor económico adicionado (EVA); valor de mercado agregado (VMA); desempenho financeiro; análise financeira; indicadores financeiros de eficiência, efetividade e eficácia; indutores do EVA; indústria automotiva

\section{Introducción}

La industria automotriz es considerada por el gobierno de Colombia como un sector de clase mundial (Proexport Colombia, 2012, p. 2), con el que se espera, en un futuro, dar impulso al crecimiento económico del país (ANDI, Cámara de la Industria Automotriz de la ANDI, CINAU, s.f. $)^{1}$. Como indica el presidente de Colombia Juan Manuel Santos: "Colombia tiene todas las condiciones para ser un jugador importante en la producción mundial de vehículos y el país puede tener una industria automotriz sólida y eficiente, que genere empleos de alta calidad" (Procolombia, 2016, p. 1). Por lo anterior, se considera de gran importancia para la comunidad empresarial, estatal y académica conocer el desempeño financiero de este sector en los últimos años, para determinar las fortalezas y debilidades financieras a las que se enfrenta para poder generar valor económico. De esta manera, se realiza un análisis financiero de este sector para el período 2008-2014,

1 La ANDI es la Asociación Nacional de Empresarios de Colombia. utilizando información contable y de mercado, que permita evaluar su crecimiento, la eficiencia en el uso de los activos, la eficacia en la gestión operativa y financiera, la efectividad para generar utilidad sobre la inversión y, además, saber si ha cumplido el propósito financiero de crear valor económico.

Los resultados muestran que las ventas y activos crecieron a partir de 2010, con un ajuste en 2013; pese a los retos que se afrontaron por la firma de nuevos tratados de libre comercio, y las crisis mundial y andina, logran rentabilidades contables, y el comportamiento de esta efectividad se explica por tres motivos: primero por la eficacia, segundo por el apalancamiento financiero y tercero por la eficiencia.

No obstante, el sector automotor en Colombia solo logró crear EVA en un año y dejó como saldo un VMA negativo en el septenio. Además, su comportamiento estuvo más relacionado con la tendencia de la rentabilidad de activo neto operacional que con la del costo de capital medio ponderado; y la primera estuvo condicionada tanto a la eficacia en el control de costos y gastos, como a la eficiencia en el uso de los activos netos operacionales. Estos hallazgos se contrastaron con datos de otras fuentes del país, con datos de Estados Unidos de América y se cotejaron con otros estudios realizados en subsectores afines como los de ensamble y autopartes en Colombia.

El artículo se estructura de la siguiente manera: en primer lugar, se plantea la metodología y se definen los indicadores financieros apropiados para este estudio; seguidamente, se muestran los principales resultados de investigaciones referentes en el ámbito nacional 
e internacional; luego se introduce una breve identificación del sector automotor en Colombia; después se analizan los indicadores que evalúan el desempeño financiero de este sector en el septenio; para finalizar se comparan y analizan estos resultados con los reportados en fuentes referentes; y se concluye sobre los principales hallazgos de esta investigación.

\section{Metodología}

Para realizar el análisis financiero del sector automotor se evaluaron el crecimiento, la eficiencia, la eficacia, la efectividad y el valor económico agregado en el período 2008-2014, siguiendo como metodología el análisis de los indicadores financieros con base en información contable y los relacionados con la gestión de valor económico. Los indicadores contables seleccionados son los de crecimiento, eficiencia, eficacia y efectividad, y como indicador de gestión del valor se calcula el valor económico agregado, EVA, y el valor de mercado agregado, VMA.

- Los indicadores de crecimiento miden el desarrollo del sector en relación con las ventas, activos y utilidad neta.

- Los indicadores de eficiencia miden la intensidad con que las empresas utilizan sus activos para generar ventas (Wild, Subramanyam \& Halsey, 2007, p. 432); para ello se calcularon los índices de rotación de los activos totales, de los activos fijos, de los activos operacionales, de los inventarios y de la cartera (Rivera-Godoy, 2004, pp. 35-36).

- Los indicadores de eficacia permiten medir de qué manera el sector logra mayores utilidades mediante la administración de los costos y gastos en las empresas. Se calcularon los márgenes de utilidad bruta, operacional, operacional después de impuestos y neta (Rivera-Godoy, 2004, p. 37).

- Los indicadores de efectividad miden la utilidad que produce la inversión; esto va a depender de la eficiencia de la gestión de los activos, de la eficacia en la administración de los costos y gastos, y del apalancamiento financiero. Su evaluación se logra mediante la revisión del rendimiento del activo (ROA) y el rendimiento del patrimonio (ROE). Con el sistema DuPont, el ROA puede descomponerse en dos factores: la rotación de activos y el margen operacional. Mientras que con el sistema DuPont ampliado, el ROE puede descomponerse en tres factores: la rotación de activos, el margen neto y el apalancamiento financiero ${ }^{2}$ (Rivera-Godoy, 2004, pp. 38, 52; Ross, Westerfield \& Jordan, 2014, pp. 62-63).

- El EVA indica la utilidad residual que queda al restar de la utilidad operacional después de impuestos y un cargo por la utilización del capital (Stewart, 2000, p. 164), tal como se expresa en la ecuación (1):

$E V A_{t}=U O D I_{t}-$ Cargo de capital ${ }_{t}$

donde

UODI $_{t}$ es la utilidad después de impuestos en el período $t$ y el cargo de capital se calcula como:

Cargo de capital $=\left(\mathrm{ANO}_{t-1}\right)\left(\mathrm{Ko}_{t}\right)$

2 Apalancamiento financiero = activo total bruto promedio /patrimonio promedio. También conocido como multiplicador del capital. 
Donde $\mathrm{ANO}_{t-1}$ es el activo neto operacional poseído al principio del período. La variable $\mathrm{ANO}_{t-1}$ se calcula a partir del capital de trabajo neto operativo $\mathrm{KTNO}_{t-1}$ y del activo fijo neto operacional $A F N O_{t-1}$, así:

$\mathrm{ANO}_{t-1}=\mathrm{KTNO}_{t-1}+\mathrm{AFNO}_{t-1}$

El KTNO ${ }_{t-1}$ se calcula restando los activos corrientes con los pasivos corrientes sin costo explícito. La variable $A_{F N O}$ se calcula restando los activos fijos operacionales con su depreciación. La variable de $\mathrm{Ko}_{t}$ es el costo de capital medio ponderado del período $t$, y según Franco Modigliani y Merton Miller (1963, p. 441) se calcula de la siguiente manera:

$K o_{t}=K e(1-L)+K i(1-t) L$

Donde Ki es el costo de la deuda, pero dado que los montos de intereses son deducibles de la base gravable de la empresa, el costo de la deuda después de impuestos queda expresado como Ki $(1-t)$. La variable $\mathrm{Ke}$ es el costo del capital propio o costo de oportunidad. La $t$ representa la tasa de impuestos de la empresa y la variable $L$ significa el nivel de endeudamiento, es decir, la división entre la deuda con costo explícito y el activo neto operacional, ANO.

Dada la complejidad para determinar Ke en las empresas que no cotizan en la bolsa de valores, se seguirá la metodología del Capital Asset Pricing Model, CAPM, de Pure Play explicada por Jorge Alberto Rivera-Godoy y Diana Stella Alarcón-Morales (2012, pp. 89-90). Bennet Stewart (2000, p. 163) presenta una segunda forma de calcular el EVA que se expone a continuación:

$E V A=\left(A N O_{t-1}\right)\left[\left(U_{A I I}\right)(1-t) /\left(\mathrm{ANO}_{t-1}\right)-\left(\mathrm{Ko}_{t}\right)\right]$

donde $\left(\mathrm{UAII}_{t}\right)(1-t) / \mathrm{ANO}_{t-1}$ es el rendimiento después de impuestos del activo neto operacional. A la diferencia entre el rendimiento después de impuestos del activo neto operacional ANO y el costo de capital medio ponderado $\mathrm{Ko}_{\mathrm{t}}$, se le conoce como el porcentaje de utilidad o pérdida residual, que es otro inductor que se obtiene con la ecuación UODI / $\left(\mathrm{ANO}_{t-1}-\mathrm{Ko}_{\mathrm{t}}\right)$.

$\mathrm{Al}$ traer a valor presente el EVA de varios años, se obtiene el valor de mercado agregado VMA del período evaluado, que se calcula de la siguiente forma:

$$
V M A=\sum_{j=1}^{j=n} \frac{E V A_{j}}{\left(1+K o_{j}\right)^{j}}
$$

También se calculó el EVA que genera el capital propio, mediante la siguiente ecuación:

EVA de capital propio ${ }_{t}=\left(\right.$ Patrimonio $\left._{t-1}\right)($ ROE $-K E)$

Para el análisis de la gestión del valor se toman en cuenta el EVA y sus cuatro inductores: utilidad operacional después de impuestos UODI, ANO, Ko y el porcentaje de utilidad o pérdida residual. También se calculan el VMA y el EVA de capital propio. Adicionalmente, se examina el EVA que genera cada unidad monetaria del ANO que se calcula dividiendo el valor 
económico agregado EVA con los activos netos operacionales ANO, mientras que la utilidad operativa después de impuestos que se requiere para crear una unidad monetaria de valor económico agregado EVA se calcula dividiendo la utilidad operativa después de impuestos UODI y el valor económico agregado EVA.

\section{Marco de referencia}

Jorge Alberto Rivera-Godoy y Ana Milena Padilla-Ospina (2015) analizan el desempeño fi- nanciero del sector de ensamble para el período 2008-2013, sobre una muestra promedio de ocho empresas, y encuentran un decrecimiento en las ventas, los activos y la utilidad neta; además, su efectividad, medida por el ROA y el ROE, ha oscilado durante el período, influenciada en gran medida por los cambios de los márgenes de utilidad (más concretamente por el costo de las ventas), y en menor proporción por los cambios en la rotación de activos y del apalancamiento financiero, como se aprecia en la tabla 1.

\begin{tabular}{|c|c|c|c|c|c|c|c|}
\hline & 2008 & 2009 & 2010 & 2011 & 2012 & 2013 & Promedio \\
\hline Ventas (MM\$) & 894.417 & 540.422 & 679.968 & 821.836 & 763.594 & 750.701 & 741.823 \\
\hline Activos (MM\$) & 503.706 & 362.558 & 348.322 & 396.030 & 389.325 & 388.727 & 398.111 \\
\hline Utilidad neta (MM\$) & 38.199 & $(832)$ & 34.919 & 11.014 & 19.768 & 8.264 & 18.555 \\
\hline \multicolumn{8}{|c|}{ ROA: Sistema DuPont } \\
\hline ROA (\%) & 8,4 & 2,7 & 15,5 & 8,5 & 7,3 & 4,4 & 7,6 \\
\hline Margen de UODI (\%) & 4,7 & 2,1 & 8,1 & 3,9 & 3,7 & 2,3 & 4,2 \\
\hline $\begin{array}{l}\text { Rotación de activos totales } \\
\text { (veces) }\end{array}$ & 1,8 & 1,2 & 1,9 & 2,2 & 1,9 & 1,9 & 1,8 \\
\hline \multicolumn{8}{|c|}{ ROE: Sistema DuPont ampliado } \\
\hline ROE & 15,4 & $-0,4$ & 19,6 & 6,3 & 11,4 & 4,6 & 9,6 \\
\hline Margen de utilidad neta (\%) & 4,3 & $-0,2$ & 5,1 & 1,3 & 2,6 & 1,1 & 2,5 \\
\hline $\begin{array}{l}\text { Rotación de activos totales } \\
\text { (veces) }\end{array}$ & 1,8 & 1,2 & 1,9 & 2,2 & 1,9 & 1,9 & 1,8 \\
\hline Apalancamiento financiero (\%) & 202,7 & 206,2 & 200,0 & 211,8 & 226,9 & 218,4 & 210,3 \\
\hline \multicolumn{8}{|c|}{ Otros indicadores de eficiencia } \\
\hline $\begin{array}{l}\text { Rotación de activos operacionales } \\
\text { (veces) }\end{array}$ & 4,4 & 3,1 & 4,8 & 4,5 & 3,4 & 3,3 & 3,9 \\
\hline Rotación de cartera (veces) & 30,5 & 20,2 & 25,9 & 26,5 & 25,4 & 27,7 & 26,0 \\
\hline Rotación de inventario (veces) & 5,8 & 4,7 & 7,4 & 6,3 & 4,2 & 4,4 & 5,3 \\
\hline Rotación de activos fijos (veces) & 19,1 & 11,3 & 15,9 & 20,6 & 16,5 & 14,2 & 16,1 \\
\hline \multicolumn{8}{|c|}{ Otros indicadores de eficacia } \\
\hline $\begin{array}{l}\text { Margen de utilidad operacional } \\
(\%)\end{array}$ & 7,3 & 3,2 & 9,7 & 4,7 & 4,4 & 3,6 & 5,6 \\
\hline Margen de utilidad bruta (\%) & 17,2 & 14,7 & 19,9 & 14,5 & 15,9 & 14,9 & 16,2 \\
\hline
\end{tabular}

\section{Tabla 1}

Indicadores del sector ensamble en Colombia

Fuente: elaboración propia, con base en Jorge Alberto Rivera-Godoy y Ana Milena Padilla-Ospina (2015, tablas 5, 6, 7 y 8, pp. 235, 236 y 238)

MM\$ = cifras en millones de pesos colombianos 
También hallaron que este sector generó un VMA en el período, a pesar de que en tres años destruyó EVA (2008, 2009 y 2013). Esta tendencia del EVA — ascender en el primer trienio y descender en los tres años siguientes-se debe a los altibajos sincrónicos de la UODI y el cargo de capital en casi todos los años, aunque con variaciones más tenues en los segundos. En la tabla 2 se muestran en detalle los principales indicadores de los inductores del EVA.

\begin{tabular}{|c|c|c|c|c|c|c|}
\hline & 2008 & 2009 & 2010 & 2011 & 2012 & 2013 \\
\hline EVA $(\mathrm{MM} \$)$ & $(13.571,0)$ & $(5.953,0)$ & $34.262,0$ & $10.736,0$ & $3.806,0$ & $(3.490,0)$ \\
\hline UODI (MM\$) & $42.349,0$ & $11.547,0$ & $54.946,0$ & $31.701,0$ & $28.606,0$ & $17.168,0$ \\
\hline Cargo de capital (MM\$) & $55.920,0$ & $17.501,0$ & $20.684,0$ & $20.966,0$ & $24.800,0$ & $20.657,0$ \\
\hline ANO (MM\$) & $298.006,0$ & $190.344,0$ & $175.484,0$ & $176.085,0$ & $184.470,0$ & $181.049,0$ \\
\hline Кo (\%) & 18,8 & 9,2 & 11,8 & 11,9 & 13,4 & 11,4 \\
\hline \multicolumn{7}{|c|}{ Indicadores del inductor UODI } \\
\hline UAII (MM\$) & $64.867,00$ & $17.513,00$ & $66.160,00$ & $38.944,00$ & $33.313,00$ & $26.790,00$ \\
\hline Impuestos (MM\$) & $22.518,00$ & $5.966,00$ & $11.214,00$ & $7.243,00$ & $4.707,00$ & $9.623,00$ \\
\hline Impuestos/UAII (\%) & 34,71 & 34,06 & 16,95 & 18,60 & 14,13 & 35,92 \\
\hline Utilidad bruta (MM\$) & $153.680,00$ & $79.266,00$ & $135.618,00$ & $119.079,00$ & $121.378,00$ & $111.739,00$ \\
\hline Costo de venta (MM\$) & $740.737,00$ & $461.155,00$ & $544.350,00$ & $702.757,00$ & $642.216,00$ & $638.962,00$ \\
\hline $\begin{array}{l}\text { Gasto de administración } \\
(\mathrm{MM} \$)\end{array}$ & $16.985,00$ & $13.499,00$ & $15.573,00$ & $18.154,00$ & $16.407,00$ & $14.933,00$ \\
\hline Gasto de venta $(\mathrm{MM} \$)$ & $71.827,00$ & $48.254,00$ & $53.885,00$ & $61.981,00$ & $71.657,00$ & $70.016,00$ \\
\hline \multicolumn{7}{|c|}{ Indicadores del inductor ANO } \\
\hline KTNO (MM\$) & 214.688 & 109.903 & 114.466 & 108.061 & 96.921 & 74.567 \\
\hline AFNO (MM\$) & 83.318 & 80.442 & 61.018 & 68.024 & 87.549 & 106.482 \\
\hline \multicolumn{7}{|c|}{ Indicadores del inductor Ko } \\
\hline $\operatorname{Ke}(\%)$ & 21,8 & 10,5 & 12,6 & 13,0 & 14,7 & 12,7 \\
\hline $\mathrm{Ki}(\%)$ & 16,0 & 7,6 & 6,6 & 9,0 & 8,6 & 7,0 \\
\hline $\begin{array}{l}\text { Deuda con costo explícito } \\
(\mathrm{MM} \$)\end{array}$ & $79.801,0$ & $45.068,0$ & $19.951,0$ & $33.834,0$ & $31.595,0$ & $28.347,0$ \\
\hline $\mathrm{L}(\%)$ & 26,8 & 23,7 & 11,4 & 19,2 & 17,1 & 15,7 \\
\hline $\mathrm{t}(\%)$ & 34,7 & 34,1 & 16,9 & 18,6 & 14,1 & 35,9 \\
\hline \multicolumn{7}{|c|}{ Sistema DuPont de la relación UODI/ANO } \\
\hline UODI/ANO (\%) & 14,21 & 6,07 & 31,31 & 18,00 & 15,51 & 9,48 \\
\hline UODI/ventas (\%) & 4,70 & 2,10 & 8,10 & 3,90 & 3,70 & 2,30 \\
\hline Ventas/ANO (veces) & 3,00 & 2,80 & 3,90 & 4,70 & 4,10 & 4,10 \\
\hline \multicolumn{7}{|c|}{ Valor del mercado agregado } \\
\hline VMA a 1-1-2008 (MM\$) & 14.600 & & & & & \\
\hline
\end{tabular}

Tabla 2

EVA y sus inductores: promedio por empresa del sector ensamble en Colombia

Fuente: Jorge Alberto Rivera-Godoy y Ana Milena Padilla-Ospina (2015, tabla 9, p. 239)

MM\$ = cifras en millones de pesos colombianos 
Jorge Alberto Rivera-Godoy y Ana Milena Padilla-Ospina (2016) realizaron un diagnóstico financiero del sector de autopartes, durante el período 2008-2014, con una muestra promedio de 68 empresas, en el cual hallaron un crecimiento continuo de sus activos y un aumento con fluctuaciones en las ventas y la utilidad neta; y que su efectividad en el logro de utilidades sobre el capital obedece, en orden de importancia, a los siguientes factores: 1 . Margen de utilidad neta; 2. Apalancamiento financiero; y 3. Rotación de activos totales, con lo que deducen que el desempeño financiero de este sector está muy influenciado por la eficacia en la gestión de los costos de venta y los gastos de administración y venta; y que su segundo factor relevante, que está relacionado con la gestión de su estructura de capital, se ha visto reducido por la tendencia a disminuir el apalancamiento financiero a lo largo del septenio. Mientras que la eficiencia en el uso de los activos totales ha sido el factor de menor impacto; aunque se presenta una mejora de la rotación de algunos activos operacionales en el último quinquenio (tabla 3).

\begin{tabular}{|c|c|c|c|c|c|c|c|c|}
\hline & 2008 & 2009 & 2010 & 2011 & 2012 & 2013 & 2014 & Promedio \\
\hline Ventas (MM\$) & $30.532,0$ & $29.936,0$ & $34.153,0$ & $38.365,0$ & $40.924,0$ & $37.288,0$ & $42.037,0$ & $36.176,0$ \\
\hline Activos (MM\$) & $28.580,0$ & $31.157,0$ & $31.303,0$ & $35.947,0$ & $37.906,0$ & $40.080,0$ & $44.444,0$ & $35.631,0$ \\
\hline Utilidad neta (MM\$) & $1.809,0$ & $1.233,0$ & $2.313,0$ & $2.656,0$ & $1.982,0$ & $1.768,0$ & $2.195,0$ & $1.994,0$ \\
\hline $\mathrm{ROA}(\%)$ & 6,5 & 4,8 & 5,8 & 5,0 & 4,0 & 2,1 & 3,3 & 4,5 \\
\hline \multicolumn{9}{|c|}{ Sistema DuPont } \\
\hline $\begin{array}{l}\text { Margen de utilidad } \\
\text { operacional después de } \\
\text { impuestos (\%) }\end{array}$ & 6,1 & 4,9 & 5,3 & 4,5 & 3,7 & 2,3 & 3,4 & 4,3 \\
\hline $\begin{array}{l}\text { Rotación de activos } \\
\text { totales (veces) }\end{array}$ & 1,1 & 1,0 & 1,1 & 1,1 & 1,1 & 1,0 & 1,0 & 1,05 \\
\hline \multicolumn{9}{|c|}{ Sistema DuPont ampliado } \\
\hline $\mathrm{ROE}(\%)$ & 11,3 & 7,1 & 12,3 & 13,2 & 8,8 & 7,3 & 8,6 & 9,8 \\
\hline \multicolumn{9}{|l|}{ Sistema DuPont } \\
\hline $\begin{array}{l}\text { Margen de utilidad } \\
\text { neta (\%) }\end{array}$ & 5,9 & 4,1 & 6,8 & 6,9 & 4,8 & 4,7 & 5,2 & 5,5 \\
\hline $\begin{array}{l}\text { Rotación de activos } \\
\text { totales (veces) }\end{array}$ & 1,1 & 1,0 & 1,1 & 1,1 & 1,1 & 1,0 & 1,0 & 1,1 \\
\hline $\begin{array}{l}\text { Apalancamiento } \\
\text { financiero (\%) }\end{array}$ & 178,5 & 171,9 & 167,2 & 168,2 & 164,1 & 162,0 & 165,4 & 168,2 \\
\hline \multicolumn{9}{|c|}{ Otros indicadores de eficiencia } \\
\hline $\begin{array}{l}\text { Rotación de activos } \\
\text { operacionales (veces) }\end{array}$ & 1,7 & 1,7 & 2,0 & 2,2 & 2,3 & 1,9 & 2,1 & 2,0 \\
\hline $\begin{array}{l}\text { Rotación de cartera } \\
\text { (veces) }\end{array}$ & 5,0 & 4,8 & 5,5 & 6,7 & 7,3 & 6,5 & 7,0 & 6,1 \\
\hline $\begin{array}{l}\text { Rotación de inventario } \\
\text { (veces) }\end{array}$ & 3,0 & 3,2 & 3,6 & 3,7 & 3,6 & 3,0 & 3,2 & 3,3 \\
\hline $\begin{array}{l}\text { Rotación de activos fijos } \\
\text { (veces) }\end{array}$ & 8,1 & 7,8 & 8,2 & 9,8 & 10,6 & 8,4 & 9,4 & 8,9 \\
\hline
\end{tabular}




\begin{tabular}{lcccccccc}
\hline & 2008 & 2009 & 2010 & 2011 & 2012 & 2013 & 2014 & Promedio \\
\hline \multicolumn{7}{c}{ Otros indicadores de eficacia } \\
\hline $\begin{array}{l}\text { Margen de utilidad } \\
\text { operacional (\%) }\end{array}$ & 8,5 & 6,8 & 8,3 & 7,9 & 6,3 & 4,9 & 6,1 & 6,9 \\
$\begin{array}{l}\text { Margen de utilidad } \\
\text { bruta (\%) }\end{array}$ & 26,0 & 24,7 & 26,1 & 24,8 & 24,4 & 24 & 24,2 & 24,9 \\
\hline
\end{tabular}

Tabla 3

Indicadores del sector autopartes en Colombia

Fuente: elaboración propia, con base en Jorge Alberto Rivera-Godoy y Ana Milena Padilla-Ospina (2016, tablas 4, 5, 6 y 7, pp. 19 y 20)

MM\$ = cifras en millones de pesos colombianos

Jorge Alberto Rivera-Godoy y Ana Milena Padilla-Ospina (2016) sugieren que, aunque el sector autopartes tuvo utilidades contables en el septenio, su EVA fue negativo en cada uno de los años, debido a que las utilidades operacionales después de impuestos no fueron suficientes para compensar el cargo de capital. "La tendencia fue a destruir menos valor con el paso de los años, en el primer trienio, y a aumentar de forma gradual la destrucción de valor, en el último cuatrienio" (p. 17). Este comportamiento del EVA, como el de sus inductores, se observa en la tabla 4.

\begin{tabular}{|c|c|c|c|c|c|c|c|}
\hline & 2008 & 2009 & 2010 & 2011 & 2012 & 2013 & 2014 \\
\hline EVA $(M M \$)$ & $(2.057,0)$ & $(731,0)$ & $(994,0)$ & $(1.239,0)$ & $(2.018,0)$ & $(2.280,0)$ & $(2.561,0)$ \\
\hline UODI (MM\$) & $1.870,0$ & $1.452,0$ & $1.823,0$ & $1.711,0$ & $1.509,0$ & 847,0 & $1.419,0$ \\
\hline Cargo de capital (MM\$) & $3.927,0$ & $2.183,0$ & $2.817,0$ & $2.950,0$ & $3.527,0$ & $3.127,0$ & $3.980,0$ \\
\hline ANO (MM\$) & $19.853,0$ & $21.103,0$ & $20.698,0$ & $22.501,0$ & $25.237,0$ & $26.340,0$ & $27.656,0$ \\
\hline Ко (\%) & 19,8 & 10,3 & 13,6 & 13,1 & 14,0 & 11,9 & 14,4 \\
\hline \multicolumn{8}{|c|}{ Indicadores del inductor UODI } \\
\hline UAII (MM\$) & $2.585,0$ & $2.036,0$ & $2.818,0$ & $3.014,0$ & $2.580,0$ & $1.820,0$ & $2.550,0$ \\
\hline Impuestos (MM\$) & 715,0 & 584,0 & 995,0 & $1.303,0$ & $1.071,0$ & 973,0 & $1.131,0$ \\
\hline Impuestos/UAII (\%) & 27,7 & 28,7 & 35,3 & 43,2 & 41,5 & 53,5 & 44,4 \\
\hline Utilidad bruta (MM\$) & $7.940,0$ & $7.393,0$ & $8.900,0$ & $9.500,0$ & $9.970,0$ & $8.940,0$ & $10.171,0$ \\
\hline Costo de venta $(\mathrm{MM} \$)$ & $22.591,0$ & $22.543,0$ & $5.253,0$ & $28.865,0$ & $30.954,0$ & $28.348,0$ & $1.866,0$ \\
\hline Gastos de administración (MM\$) & $2.529,0$ & $2.547,0$ & $2.761,0$ & $3.060,0$ & $3.382,0$ & $3.115,0$ & $3.266,0$ \\
\hline Gastos de venta (MM\$) & $2.827,0$ & $2.810,0$ & $3.321,0$ & $3.426,0$ & $4.008,0$ & $4.005,0$ & $4.355,0$ \\
\hline \multicolumn{8}{|c|}{ Indicadores del inductor ANO } \\
\hline KTNO (MM\$) & 12.483 & 12.380 & 11.775 & 14.053 & 14.409 & 14.503 & 15.195 \\
\hline AFNO (MM\$) & 7.370 & 8.723 & 8.923 & 8.448 & 10.828 & 11.837 & 12.461 \\
\hline \multicolumn{8}{|c|}{ Indicadores del inductor Ko } \\
\hline $\operatorname{Ke}(\%)$ & 23,8 & 12,2 & 16,8 & 15,4 & 17,0 & 14,7 & 17,7 \\
\hline $\mathrm{Ki}(\%)$ & 16,0 & 7,6 & 6,6 & 9,0 & 8,6 & 7,0 & 7,7 \\
\hline Deuda con costo explícito (MM\$) & $6.546,0$ & $5.782,0$ & $5.259,0$ & $5.002,0$ & $6.370,0$ & $6.504,0$ & $6.837,0$ \\
\hline
\end{tabular}




\begin{tabular}{lrrrrrrr}
\hline & $\mathbf{2 0 0 8}$ & $\mathbf{2 0 0 9}$ & $\mathbf{2 0 1 0}$ & $\mathbf{2 0 1 1}$ & $\mathbf{2 0 1 2}$ & $\mathbf{2 0 1 3}$ & $\mathbf{2 0 1 4}$ \\
\hline $\mathrm{L}(\%)$ & 33,0 & 27,4 & 25,4 & 22,2 & 25,2 & 24,7 & 24,7 \\
$\mathrm{t}(\%)$ & 27,7 & 28,7 & 35,3 & 43,2 & 41,5 & 53,5 & 44,4 \\
\hline \multicolumn{7}{c}{ Sistema DuPont de la relación } & UODI/ANO \\
\hline UODI/ ANO (\%) & 9,4 & 6,9 & 8,8 & 7,6 & 6,0 & 3,2 & 5,1 \\
UODI/ventas (\%) & 6,1 & 4,9 & 5,3 & 4,5 & 3,7 & 2,3 & 3,4 \\
Ventas/ANO (veces) & 1,5 & 1,4 & 1,6 & 1,7 & 1,6 & 1,4 & 1,5 \\
\hline \multicolumn{7}{c}{ Valor del mercado agregado } \\
\hline VMA a 1-1-2008 (MM\$)
\end{tabular}

Tabla 4

EVA y sus inductores: promedio por empresa del sector autopartes en Colombia Fuente: Jorge Alberto Rivera-Godoy y Ana Milena Padilla-Ospina (2016, tabla 8, p. 21) MM\$ = cifras en millones de pesos colombianos

En los últimos años, la revista Dinero ha publicado cada año la lista de las 5.000 empresas más grandes de Colombia, junto con indicadores financieros que permiten evaluar su desempeño financiero. En este estudio se han escogido los datos correspondientes a una muestra promedio de 46 empresas del sector automotor en el período 2008-2014 (tabla 5).

\begin{tabular}{|c|c|c|c|c|c|c|c|c|}
\hline Indicador & 2008 & 2009 & 2010 & 2011 & 2012 & 2013 & 2014 & Promedio \\
\hline \multicolumn{9}{|c|}{ Crecimiento } \\
\hline Ventas (\$) & 169.858 & 142.100 & 177.654 & 194.254 & 234.370 & 213.974 & 229.216 & 194.490 \\
\hline Activos (\$) & 114.455 & 116.001 & 118.435 & 118.666 & 143.825 & 131.228 & 148.249 & 127.266 \\
\hline Patrimonio $(\$)$ & 57.945 & 65.822 & 65.822 & 60.470 & 77.740 & 73.849 & 78.728 & 68.625 \\
\hline Utilidad neta (\$) & 7.834 & 2.552 & 9.957 & 5.314 & 7.404 & 4.322 & 7.301 & 6.383 \\
\hline Ebitda $(\$)$ & 17.897 & 10.046 & 19.615 & 12.966 & 16.690 & 12.948 & 17.974 & 15.448 \\
\hline \multicolumn{9}{|c|}{ Eficiencia } \\
\hline $\begin{array}{l}\text { Rotación de activos } \\
\text { totales }\end{array}$ & 1,48 & 1,22 & 1,50 & 1,64 & 1,63 & 1,63 & 1,55 & 1,52 \\
\hline \multicolumn{9}{|c|}{ Eficacia } \\
\hline Ebitda/ventas (\%) & 10,5 & 7,1 & 11,0 & 6,7 & 7,1 & 6,1 & 7,8 & 8,0 \\
\hline $\begin{array}{l}\text { Utilidad neta/ventas } \\
(\%)\end{array}$ & 4,6 & 1,8 & 5,6 & 2,7 & 3,2 & 2,0 & 3,2 & 3,3 \\
\hline \multicolumn{9}{|c|}{ Endeudamiento } \\
\hline $\begin{array}{l}\text { Apalancamiento } \\
\text { financiero (\%) }\end{array}$ & 202,4 & 186,2 & 178,1 & 187,7 & 189,9 & 181,4 & 183,2 & 187,0 \\
\hline \multicolumn{9}{|c|}{ Efectividad } \\
\hline ROE (\%) & 13,9 & 4,1 & 15,0 & 8,4 & 9,8 & 6,0 & 9,0 & 9,4 \\
\hline $\begin{array}{l}\text { Número de } \\
\text { empresas }\end{array}$ & 51,0 & 48,0 & 47,0 & 50,0 & 44,0 & 47,0 & 40,0 & 46,0 \\
\hline
\end{tabular}

Tabla 5

Indicadores financieros promedio de la industria automotriz en Colombia (2008-2014)

Fuente: elaboración propia, con datos de la revista Dinero (2009, 2010, 2011, 2012, 2013, 2014 y 2015)

$\mathrm{MM} \$=$ cifras en millones de pesos colombianos 
En la parte $a$ de la tabla 5 se puede observar un aumento continuo de los activos, solo interrumpido por una caída en 2013, un crecimiento con algunos altibajos de las ventas y un comportamiento ondulante del patrimonio, la utilidad neta y la Ebitda ${ }^{3}$, y de manera sincrónica estas dos últimas.

La rotación de los activos tiende a aumentar en el período 2008-2011, para luego decrecer hasta 2014. Su pico más alto se presentó en 2011 (1,64 veces) y el más bajo en 2009 (1,22 veces); los indicadores Ebitda/ventas y utilidad neta/ventas son oscilantes y varían de manera simultánea con el cambio de la utilidad neta y la Ebitda, por ello no se pueden detectar tendencias de la eficacia en el septenio, como se aprecia en las secciones $b$ y $c$ de la tabla 5 .

El apalancamiento financiero fue en promedio de $187 \%$, con una tendencia a disminuir hasta 2010, al pasar de 202,4\% en 2008 a 178,1\% en 2010; en los siguientes dos años sube hasta llegar a 189,9\% en 2012, y después varía (parte $d$ de la tabla 5).

El rendimiento del patrimonio varía año tras año, manteniendo una relación positiva con el margen de utilidad neta, donde las crestas más alta y más baja se presentan en 2010 (15,0\%) y en 2009 (4,1\%), respectivamente, como se muestra en la parte $e$ de la tabla 5. A partir de la información extraída de la página web de Aswath Damodaran (2015) de las empresas del sector automotor de Estados Unidos de

3 Ebitda (Earnings Before Interest, Taxes, Depreciation, and Amortization), utilidad antes de interés, impuestos, depreciación y amortización.
América $^{4}$, se calculan los indicadores de desempeño financiero presentados en la tabla 6 .

Este sector destruye valor económico en tres de los siete años de estudio: 2009, 2010 y 2012. El año en que más valor creó fue 2011, con US\$56.676 millones y el año en que menos valor creó fue 2014 (US\$722 millones). Durante los años en que se destruye valor económico, la UODI disminuye considerablemente, mientras que el cargo de capital aumenta, como sucedió en 2009 y 2012. Después de que la UODI alcanza sus dos picos más altos (US\$66.390 MM en 2008 y US\$103.293 MM en 2011) tiende a reducirse en los años siguientes, conservando un comportamiento semejante a la UAII y a los impuestos. El cargo de capital fluctúa en casi todos los años al unísono con el ANO (es notable su reducción en los dos últimos años), a la vez que el Ko cambia de tendencia, por lo general, cada dos años, lo que no permite detectar una tendencia en su comportamiento en el septenio. Los mayores y menores valores de cargo de capital, presentados en 2009 (US\$53.967 MM) y 2013 (US\$15.080 MM), no coinciden con los más altos o más bajos valores del ANO y el Ko (tabla 6).

La relación UODI/ANO es siempre positiva y se comporta de manera afín a la UODI, lo que demuestra que la destrucción de valor en los años 2009, 2010 y 2012 no se da porque se hayan presentado pérdidas operacionales contables, sino porque los rendimientos de sus activos han sido inferiores a los costos de capital (sección $a$ de la tabla 6).

4 Los datos del sector automotor de Estados Unidos de América se componen de una muestra de 72 empresas para 2008, 76 para el para 2009, 64 para 2010, 63 para 2011, 66 para 2012, 101 para 2013 y 97 para 2014. 


\begin{tabular}{|c|c|c|c|c|c|c|c|}
\hline Indicador & 2008 & 2009 & 2010 & 2011 & 2012 & 2013 & 2014 \\
\hline \multicolumn{8}{|c|}{ EVA y sus inductores } \\
\hline EVA (MMUS\$) & $28.104,00$ & $(42.032,00)$ & $(36.984,00)$ & $56.676,00$ & $(3.375,00)$ & $6.508,00$ & 722,00 \\
\hline UODI (MMUS\$) & $66.390,00$ & $11.935,00$ & $2.300,00$ & $103.293,00$ & $49.321,00$ & $21.566,00$ & $16.306,00$ \\
\hline $\begin{array}{l}\text { Cargo de capital } \\
\text { (MMUS\$) }\end{array}$ & $38.285,00$ & $53.967,00$ & $39.285,00$ & $46.617,00$ & $52.696,00$ & $15.058,00$ & $15.584,00$ \\
\hline ANO (MMUS\$) & $616.692,00$ & $619.340,00$ & $531.646,00$ & $675.110,00$ & $703.684,00$ & $179.137,00$ & $276.728,00$ \\
\hline Кo (\%) & 6,21 & 8,71 & 7,39 & 6,91 & 7,49 & 8,41 & 5,63 \\
\hline UODI/ANO (\%) & 10,80 & 1,90 & 0,40 & 15,30 & 7,00 & 12,00 & 5,90 \\
\hline \multicolumn{8}{|c|}{ Indicadores del inductor UODI } \\
\hline UAII (MMUS\$) & 85.304 & 13.666 & 2.602 & 68.178 & 59.363 & 23.242 & 17.745 \\
\hline $\begin{array}{l}\text { Impuestos } \\
(\text { MMUS\$) }\end{array}$ & 18.915 & 1.731 & 302 & 15.813 & 10.042 & 1.677 & 1.439 \\
\hline \multicolumn{8}{|c|}{ Indicadores del inductor Ko } \\
\hline $\operatorname{Ke}(\%)$ & 11,31 & 11,37 & 10,79 & 11,56 & 11,81 & 9,8 & 9,0 \\
\hline Ki (\%) & 4,74 & 7,84 & 4,83 & 3,89 & 3,26 & 7,04 & 3,21 \\
\hline $\mathrm{L}(\%)$ & 66,95 & 58,10 & 48,43 & 54,17 & 47,58 & 43,13 & 46,36 \\
\hline $\mathrm{t}(\%)$ & 22,17 & 12,67 & 11,61 & 23,19 & 16,92 & 7,21 & 8,11 \\
\hline \multicolumn{8}{|c|}{ Valor del mercado agregado } \\
\hline $\begin{array}{l}\text { VMA a 1-1-2009 } \\
\text { (MMUS\$) }\end{array}$ & 3.569 & & & & & & \\
\hline \multicolumn{8}{|c|}{ EVA generado por el capital propio } \\
\hline $\begin{array}{l}\text { EVA de capital } \\
\text { propio (MMUS\$) }\end{array}$ & $6.488,0$ & $(40.526,0)$ & $(58.539,0)$ & 436,0 & $(2.263,0)$ & $9.428,0$ & $7.329,0$ \\
\hline ROE (\%) & 14,0 & $-13,3$ & $-18,8$ & 11,7 & 11,2 & 20,2 & 17,6 \\
\hline $\operatorname{Ke}(\%)$ & 11,3 & 11,4 & 10,8 & 11,6 & 11,8 & 9,8 & 9,0 \\
\hline $\begin{array}{l}\text { Patrimonio } \\
(\text { MMUS\$) }\end{array}$ & $239.879,0$ & $164.353,0$ & $197.550,0$ & $360.311,0$ & $396.773,0$ & $90.158,0$ & $85.464,0$ \\
\hline
\end{tabular}

Tabla 6

Indicadores promedio del sector automotor en Estados Unidos de América Fuente: elaboración propia a partir de datos de Aswath Damodaran (2015) MMUS\$ = cifras monetarias en millones de dólares

El Ko oscila entre 8,71\% y 5,63\%; su valor depende de la influencia del nivel de endeudamiento, el costo de la deuda y el costo del capital propio. El nivel de endeudamiento se reduce de forma continua, al pasar de 66,95\% en 2008 a 46,36\% en 2014, con ciertos repuntes en 2011 y 2014. El costo de la deuda, también, sigue una tendencia decreciente a partir de 2010, solamente interrumpido con un incremento en 2013. El costo de capital propio se mantiene entre $9 \%$ y $11,56 \%$, y presenta una reducción importante en los dos últimos años (sección $c$ de la tabla 6). 
El valor presente de los EVA generados durante los últimos siete años tiene un valor positivo de US\$3.569 MM, lo cual sugiere que este sector ha sido generador de valor económico durante este período, a pesar de que destruye valor en tres años (sección $d$ de la tabla 6).

El EVA del capital propio sigue un comportamiento similar al EVA de todos los recursos, pero con una particularidad: en los años 2009 y 2010, además de la destrucción del EVA, se presentaron pérdidas netas contables. El ROE es altamente variable en relación con el $\mathrm{Ke}$; mientras el patrimonio aumenta hasta 2012, pero se reduce de manera considerable en los dos últimos años. Esto permite inferir que la variación del EVA del capital propio está sujeta, en gran parte, al comportamiento del ROE en el septenio.

\section{La industria automotriz en Colombia}

La industria automotriz en Colombia comprende las actividades de ensamble de vehículos ligeros, buses, camiones y motocicletas, y la producción de autopartes para ensamble (Proexport Colombia, 2012). El sector automotor de Colombia representa el 1,6\% del PIB y el $4 \%$ de la producción bruta industrial, y genera más de 22.300 empleos (BBVA Research, América del Sur, Colombia, 2015). Su demanda es principalmente doméstica, sin embargo, el $70 \%$ de los autos vendidos en Colombia es importado y el $93 \%$ de las motos que se venden internamente se produce en el país (BBVA Research, América del Sur, Colombia, 2015). Hay ocho empresas que ensamblan vehículos, nueve que ensamblan motocicletas y más de cincuen- ta empresas que producen partes para el sector (ANDI, Cámara de la Industria Automotriz de la ANDI, CINAU, s.f.). Los principales destinos de las exportaciones del sector automotor y sus partes en 2014 fueron: Ecuador (41,2\%), México (32,5\%), Perú (8,5\%). Estados Unidos (3,5\%) y Venezuela (3,1\%) (BBVA Research, América del Sur, Colombia, 2015).

En Colombia, la producción de vehículos, camiones y buses nacionales representa el $32,7 \%$ del mercado automotor frente al 67,3\% que conforman los vehículos que ingresan al país procedentes de México, Corea del Sur, China, Ecuador e India (Reina, Oviedo \& Moreno, Fedesarrollo, 2014). Aunque ha habido un aumento en la producción del sector, al pasar de \$4.715.523 millones en 2008 a \$6.077.548 millones en 2012, la importación de productos ha aumentado casi al doble de la producción nacional durante el período de 2008-2013; entre tanto, las exportaciones del sector automotor se reducen casi un 50\% durante los años 2009, 2010 y 2011. En cuanto al consumo interno del sector, ha aumentado en $62 \%$ en los últimos seis años (tabla 7).

El gobierno nacional considera que el sector automotor tiene un gran potencial de desarrollo. Por esta razón se ha propuesto fortalecerlo con medidas como la atracción de inversión extranjera mediante los depósitos habilitados para transformación o ensamble (DHTE), el régimen de ensamble para la industria automotriz en el marco de la $\mathrm{CAN}^{5}$ (donde se paga el cero por ciento (0\%) de arancel en la importación de vehículos con los países suscritos), el Programa de Fomento

5 Comunidad Andina: Bolivia, Colombia, Ecuador, Perú. 


\begin{tabular}{lrrrrrr}
\hline & 2008 & \multicolumn{1}{c}{ 2009 } & 2010 & 2011 & \multicolumn{1}{c}{ 2012 } & 2013 \\
\hline Producción nacional (MM\$) & 4.715 .523 & 3.692 .175 & 4.833 .712 & 5.761 .853 & 6.077 .548 & n.d. \\
Importaciones CIF (MM\$) & 6.375 .456 & 5.100 .678 & 7.214 .541 & 11.207 .818 & 11.191 .503 & 9.466 .710 \\
Exportaciones FOB (MM\$) & 1.065 .656 & 536.641 & 655.181 & 770.704 & 1.023 .269 & 1.572 .303 \\
Consumo aparente (MM\$) & 10.025 .324 & 8.256 .211 & 11.393 .072 & 16.198 .967 & 16.245 .783 & n.d. \\
TOTAL (MM\$) & 22.181 .959 & 17.585 .705 & 24.096 .506 & 33.939 .342 & 34.538 .103 & 11.039 .013 \\
\hline
\end{tabular}

Tabla 7

Composición del mercado automotor 2008-2013

Fuente: Mauricio Reina, Sandra Oviedo y Jonathan Moreno, Fedesarrollo (2014, p. 65)

MM\$ = cifras en millones de pesos colombianos, el sector automotor incluye las actividades 3410, 3420, 3430 del CIIU (Código industrial internacional uniforme); el consumo aparente equivale a la suma entre la producción nacional y las importaciones y este resultado se resta con las exportaciones.

n.d. = no hay datos

para la Industria Automotriz, PROFIA, las zonas francas para ensamble y el convenio automotor entre Colombia y Brasil (Procolombia, 2016, p. 5). Por otra parte, el país cuenta con arancel cero (0) para la exportación de vehículos comerciales de pasajeros y carga, vehículos utilitarios y motos a países como México, Perú y Chile, y estimula la competencia en el país (Procolombia, 2016, p. 3).

No obstante, hay diversas expectativas con la suscripción el tratado de libre comercio con Corea del Sur, un país con una industria automotriz muy desarrollada, en la que se pactó la reducción de los aranceles de los carros que provienen de Corea del Sur en 3,5\% anual por un espacio de 10 años hasta lograr el 0\% de arancel. Además, de todo lo que Colombia importó de Corea del Sur en 2013, el 41\% correspondía a productos de la industria automotriz (Ministerio de Comercio, Industria y Turismo, 2014).

Sin embargo, en el país se siguen haciendo inversiones importantes para fortalecer al sector, como la inauguración de la Zona Franca Industrial Colmotores, en Cundinamarca, con una inversión de más de US\$200 millones de dólares, de la cual se espera que produzca unas 60.000 partes de automóvil al año (Ministerio de Comercio, Industria y Turismo, 2013) y un mayor desarrollo de esta industria. Además, la ensambladora Daimler Colombia SAS (Mercedes-Benz) invirtió en su planta de Funza, Cundinamarca, para el ensamble de buses del sistema masivo del país (Superintendencia de Sociedades, 2015a).

Pero contrario a la inversión realizada por General Motors Colombia, la Compañía Colombiana Automotriz (CCA), que ensambla los vehículos Mazda, cerró la fabricación de vehículos en 2014, debido a las dificultades comerciales presentadas con Venezuela y Ecuador, además de la importación de autos procedentes de México que afectó esta marca de autos (Portafolio, 2014). Esto deja entrever que el sector se puede ver afectado tanto por la competitividad de los demás países que tienen una producción menos costosa, como por los tratados de libre comercio firmados por Colombia. 


\section{Desempeño financiero de las empresas del sector automotor colombiano (año 2008-2014)}

Para el cálculo de los indicadores contables y los relacionados con la creación de valor se tomaron los estados financieros de las empresas del sector automotor colombiano con código industrial internacional uniforme (CIIU) correspondiente al ensamble de vehículos (C291), a la fabricación de partes, piezas y accesorios de autopartes para vehículos (C2930 y C2219) y a la fabricación de carrocerías para vehículos automotores (C2920) de los últimos siete años de la base de datos EMIS Benchmark (2015). De esta forma, se obtuvo información de 81 em- presas para los años 2008 y 2010, 77 empresas para los años 2009 y 2011, 74 empresas para 2012, 76 empresas para 2013 y 69 empresas para 2014. En el Anexo 1 se identifica cada una de las empresas.

\subsection{Evaluación del crecimiento}

La tendencia de las ventas y los activos es crecer durante los cinco últimos años, con un quiebre en 2013, cuando se presentaron disminuciones en ambos indicadores. Esta tendencia se inicia desde 2010, después de presentar en 2009 los más bajos valores del período analizado. Por su parte, las utilidades netas no siguen un patrón de comportamiento, sino que fluctúan año tras año (tabla 8).

\begin{tabular}{lrrrrrrrc}
\hline & $\mathbf{2 0 0 8}$ & $\mathbf{2 0 0 9}$ & $\mathbf{2 0 1 0}$ & $\mathbf{2 0 1 1}$ & $\mathbf{2 0 1 2}$ & $\mathbf{2 0 1 3}$ & $\mathbf{2 0 1 4}$ & Promedio \\
\hline Ventas (MM\$) & 105.189 & 82.973 & 105.910 & 129.940 & 138.582 & 131.158 & 134.813 & 118.366 \\
Activos (MM\$) & 69.640 & 65.541 & 66.527 & 78.035 & 86.162 & 85.955 & 92.482 & 77.763 \\
Utilidad Neta (MM\$) & 4.954 & 1.018 & 5.936 & 3.633 & 4.385 & 2.623 & 2.827 & 3.625 \\
\hline
\end{tabular}

Tabla 8

Ventas, activos y utilidad neta promedio del sector automotor en Colombia Fuente: elaboración propia con base en EMIS Benchmark (2015) MM\$ = cifras en millones de pesos colombianos

\subsection{Evaluación de la eficiencia}

En general, los indicadores de eficiencia decaen en 2009 , aumentan entre 2010 y 2011 y vuelven a reducirse entre 2012-2013. Como era de esperarse, la rotación de los activos operacionales es superior a la de los activos totales; en este caso, la duplica en casi todos los años, lo que demuestra que este sector tiene valores considerables en otros activos. La rotación de los ac- tivos fijos, inventarios y cartera mantienen, en ese orden jerárquico, una relación positiva con la rotación de activos operacionales. De estos tres activos operacionales, la cartera presenta las mayores variaciones en su rotación durante el septenio (va de 10,0 veces en 2009 a 16,5 veces en 2012), mientras que los inventarios presentan una rotación más alisada, que fluctúa sobre 4,6 veces (tabla 9). 


\begin{tabular}{lrrrrrrrr}
\hline & $\mathbf{2 0 0 8}$ & $\mathbf{2 0 0 9}$ & $\mathbf{2 0 1 0}$ & $\mathbf{2 0 1 1}$ & $\mathbf{2 0 1 2}$ & $\mathbf{2 0 1 3}$ & $\mathbf{2 0 1 4}$ & Promedio \\
\hline $\begin{array}{l}\text { Rotación de activos operacionales } \\
\text { (veces) }\end{array}$ & 3,1 & 2,6 & 3,4 & 3,6 & 3,1 & 2,8 & 3,0 & 3,1 \\
Rotación de activos totales (veces) & 1,5 & 1,2 & 1,6 & 1,8 & 1,7 & 1,5 & 1,5 & 1,6 \\
Rotación de cartera (veces) & 13,0 & 10,0 & 12,6 & 14,6 & 16,5 & 14,9 & 14,4 & 13,7 \\
Rotación de inventario (veces) & 4,7 & 4,4 & 5,8 & 5,4 & 4,3 & 3,9 & 4,0 & 4,6 \\
Rotación de activos fijos (veces) & 14,0 & 10,3 & 12,8 & 16,2 & 15,0 & 12,0 & 12,3 & 13,2 \\
\hline
\end{tabular}

Tabla 9

Indicadores de eficiencia

Fuente: elaboración propia, con base en EMIS Benchmark (2015)

\subsection{Evaluación de la eficacia}

En la tabla 10 se puede observar que en términos generales todos los indicadores de eficacia presentan una variabilidad de aumento-disminución en cada uno de los años; logran los mejores resultados en 2010 y los peores índices en 2013, excepto el margen neto que tuvo su gran baja en 2009. A pesar de que no se nota una tendencia, se mantienen sobre unos valores positivos, lo que indica que no se ha caído en pérdidas contables. El promedio del margen bruto
$(18,4 \%)$ y el de la utilidad operacional $(5,8 \%)$ muestran que los costos de ventas $(81,6 \%)$ y los gastos de administración y venta (12,6\%), en su orden, son las dos erogaciones que más influyen en la gestión eficaz del sector; mientras que la diferencia entre el margen de utilidad operacional y el margen de utilidad neta, que es en promedio del 2,7\%, demuestra que los impuestos $(1,7 \%)$ y los otros ingresos (y gastos) no operacionales $(1,0 \%)$, aunque importantes, no son tan significativos como los primeros.

\begin{tabular}{lcrrrrrrr}
\hline & $\mathbf{2 0 0 8}$ & $\mathbf{2 0 0 9}$ & $\mathbf{2 0 1 0}$ & $\mathbf{2 0 1 1}$ & $\mathbf{2 0 1 2}$ & $\mathbf{2 0 1 3}$ & $\mathbf{2 0 1 4}$ & Promedio \\
\hline Margen de utilidad bruta (\%) & 19,5 & 17,9 & 21,7 & 17,2 & 18,1 & 17,1 & 17,0 & 18,4 \\
Margen de utilidad operacional (\%) & 7,6 & 4,4 & 9,3 & 5,6 & 4,9 & 3,9 & 4,8 & 5,8 \\
Margen de utilidad operacional & & & & & & & & \\
después de impuestos (\%) & 5,1 & & 7,3 & 4,0 & 3,7 & 2,3 & 3,3 & 4,1 \\
Margen de utilidad neta (\%) & 4,7 & 1,2 & 5,6 & 2,8 & 3,2 & 2,0 & 2,1 & 3,1 \\
\hline
\end{tabular}

Tabla 10

Indicadores de eficacia

Fuente: elaboración propia, con base en EMIS Benchmark (2015)

\subsection{Evaluación de la efectividad}

El ROA promedio del septenio fue de 6,4\%; es el resultado de la combinación entre el margen UODI $(4,1 \%)$ y la rotación de activos totales (1,6 veces). El comportamiento del ROA fue muy similar al del margen UODI y presentó su pico más alto en 2010, luego descendió en los tres años siguientes, para subir en 2014; pero con una relación con la rotación de activos menos alta (tabla 11). 


\section{Promedio} ROA: Sistema DuPont

\begin{tabular}{|c|c|c|c|c|c|c|c|c|}
\hline ROA (\%) & 7,7 & 3,7 & 11,7 & 7,2 & 6,3 & 3,5 & 5,0 & 6,4 \\
\hline $\begin{array}{l}\text { Margen de utilidad operacional } \\
\text { después de impuestos (\%) }\end{array}$ & 5,1 & 3,0 & 7,3 & 4,0 & 3,7 & 2,3 & 3,3 & 4,1 \\
\hline Rotación de activos totales (veces) & 1,5 & 1,2 & 1,6 & 1,8 & 1,7 & 1,5 & 1,5 & 1,6 \\
\hline \multicolumn{9}{|c|}{ ROE: Sistema DuPont Ampliado } \\
\hline ROE (\%) & 13,7 & 2,9 & 16,6 & 9,6 & 10,6 & 5,9 & 6,1 & 9,3 \\
\hline Margen de utilidad neta (\%) & 4,7 & 1,2 & 5,6 & 2,8 & 3,2 & 2,0 & 2,1 & 3,1 \\
\hline Rotación de activos totales (veces) & 1,5 & 1,2 & 1,6 & 1,8 & 1,7 & 1,5 & 1,5 & 1,6 \\
\hline Apalancamiento financiero (\%) & 192,4 & 190,9 & 184,3 & 191,4 & 197,6 & 192,9 & 193,3 & 191,8 \\
\hline
\end{tabular}

Tabla 11

Indicadores de efectividad

Fuente: elaboración propia, con base en EMIS Benchmark (2015)

Además, se observa que la rotación de los activos es menos variable que el margen de UODI: la rotación oscila entre 1,6 y 1,8 veces (no teniendo en cuenta 2009); mientras que el margen fluctúa entre 2,3\% y 7,3\%. Esta variabilidad es más afín con el ROA, con lo que se puede deducir que el comportamiento de la efectividad para rentabilizar los activos por parte del sector automotor tiene una mayor influencia de la eficacia en el control de costos y gastos (en especial los costos de la venta), que en la eficiencia en el uso de sus activos.

Por su parte, el ROE promedio fue de 9,3\%, que se deriva del producto de los siguientes indicadores: el margen de utilidad neta $(3,1 \%)$, la rotación de activos totales (1,6 veces) y el apalancamiento financiero (191,8\%). Durante el septenio, el ROE presenta altibajos y mantiene una mayor relación positiva con el margen neto que con los otros factores influyentes como la rotación de activos y el apalancamiento financiero (tabla 11).
Además, la intensidad de su variabilidad (de $2,9 \%$ a $16,6 \%$ ) se ajusta más al comportamiento del margen neto que oscila entre $1,2 \%$ y $5,6 \%$ que al cambio del apalancamiento financiero, que va de 190,9\% a 197,6\% (excluyendo 2010), y a la reducida variación de la rotación de activos, comentada en el párrafo anterior. Esto significa que el comportamiento de la efectividad de este sector obedece en orden de importancia a: 1. Eficacia. 2. Apalancamiento financiero y 3. Eficiencia.

El hecho de que el ROE sea mayor que el ROA, excepto en 2009, y que estos sean positivos durante todo el septenio, es una evidencia de que este sector se benefició del apalancamiento financiero, a pesar de que no siguió un patrón de endeudamiento después de los tres primeros años, cuando se nota una reducción continua. 


\subsection{Evaluación del valor económico agregado del sector automotor, período 2008-2014}

La empresa promedio del sector automotor colombiano solo ha generado valor económico agregado en un año (2010), de los siete años de estudio. En los tres primeros años, la tenden- cia era favorable pues se pasó a destruir menos valor en 2009, hasta crear valor en 2010; pero en el siguiente trienio se fue incrementando la destrucción de valor, y solo en 2014 se revirtió esta tendencia, pero se mantuvo un EVA negativo (tabla 12).

\begin{tabular}{|c|c|c|c|c|c|c|c|}
\hline & 2008 & 2009 & 2010 & 2011 & 2012 & 2013 & 2014 \\
\hline EVA (MM\$) & $(3.187,0)$ & $(1.522,0)$ & $2.410,0$ & $(524,0)$ & $(2.166,0)$ & $(3.061,0)$ & $(2.745,0)$ \\
\hline UODI (MM\$) & $5.368,0$ & $2.501,0$ & $7.726,0$ & $5.216,0$ & $5.171,0$ & $2.994,0$ & $4.500,0$ \\
\hline Cargo de capital (MM\$) & $8.555,0$ & $4.022,0$ & $5.315,0$ & $5.741,0$ & $7.337,0$ & $6.055,0$ & $7.246,0$ \\
\hline ANO (MM\$) & $43.891,0$ & $38.640,0$ & $37.896,0$ & $40.452,0$ & $46.755,0$ & $46.696,0$ & $46.940,0$ \\
\hline Кo (\%) & 19,5 & 10,4 & 14,0 & 14,2 & 15,7 & 13,0 & 15,4 \\
\hline \multicolumn{8}{|c|}{ Indicadores del inductor UODI } \\
\hline UAII (MM\$) & $7.967,0$ & $3.644,0$ & $9.856,0$ & $7.214,0$ & $6.733,0$ & $5.106,0$ & $6.407,0$ \\
\hline Impuestos (MM\$) & $2.599,0$ & $1.143,0$ & $2.130,0$ & $1.997,0$ & $1.562,0$ & $2.111,0$ & $1.907,0$ \\
\hline Impuestos/UAII (\%) & 32,6 & 31,4 & 21,6 & 27,7 & 23,2 & 41,3 & 29,8 \\
\hline Utilidad bruta (MM\$) & $20.535,0$ & $14.860,0$ & $22.980,0$ & $22.308,0$ & $25.025,0$ & $22.466,0$ & $22.903,0$ \\
\hline Costo de venta (MM\$) & $84.653,0$ & $68.113,0$ & $82.930,0$ & $107.632,0$ & $113.557,0$ & $108.692,0$ & $99.493,0$ \\
\hline Gastos de administración (MM\$) & $3.778,0$ & $3.685,0$ & $4.185,0$ & $4.824,0$ & $5.142,0$ & $4.670,0$ & $5.255,0$ \\
\hline Gastos de venta (MM\$) & $8.790,0$ & $7.531,0$ & $8.939,0$ & $10.270,0$ & $13.150,0$ & $12.691,0$ & $11.241,0$ \\
\hline \multicolumn{8}{|c|}{ Indicadores del inductor ANO } \\
\hline KTNO (MM\$) & 29.957 & 22.512 & 23.185 & 25.041 & 25.559 & 22.406 & 21.984 \\
\hline AFNO (MM\$) & 13.934 & 16.127 & 14.711 & 15.411 & 21.196 & 24.290 & 24.956 \\
\hline \multicolumn{8}{|c|}{ Indicadores del Inductor Ko } \\
\hline $\operatorname{Ke}(\%)$ & 23,1 & 12,2 & 16,0 & 16,2 & 18,1 & 15,2 & 18,0 \\
\hline $\mathrm{Ki}(\%)$ & 16,0 & 7,6 & 6,6 & 9,0 & 8,6 & 7,0 & 7,7 \\
\hline Deuda con costo explícito (MM\$) & $12.877,0$ & $9.864,0$ & $6.891,0$ & $8.372,0$ & $9.779,0$ & $9.378,0$ & $9.559,0$ \\
\hline $\mathrm{L}(\%)$ & 29,3 & 25,5 & 18,2 & 20,7 & 20,9 & 20,1 & 20,4 \\
\hline $\mathrm{t}(\%)$ & 33,0 & 31,0 & 22,0 & 28,0 & 23,0 & 41,0 & 30,0 \\
\hline \multicolumn{8}{|c|}{ Sistema DuPont del índice UODI/ANO } \\
\hline UODI/ ANO (\%) & 12,2 & 6,4 & 20,3 & 12,9 & 11,0 & 6,4 & 9,6 \\
\hline UODI/ventas (\%) & 5,1 & 3,0 & 7,3 & 4,0 & 3,7 & 2,3 & 3,3 \\
\hline Ventas/ANO (veces) & 2,4 & 2,1 & 2,8 & 3,2 & 3,0 & 2,8 & 2,9 \\
\hline \multicolumn{8}{|c|}{ Valor del mercado agregado } \\
\hline VMA a 1-1-2008 (MM\$) & $(6.036)$ & & & & & & \\
\hline
\end{tabular}

Tabla 12

EVA promedio por empresa y sus inductores

Fuente: elaboración propia, con base en EMIS Benchmark (2015), Superintendencia Financiera (2015b) y Aswath Damoda$\operatorname{ran}(2015)$

MM\$ = cifras en millones de pesos colombianos 
La tendencia del EVA depende de los indicadores UODI y del cargo de capital, pero al analizar sus relaciones con el EVA, se observa que aquella positiva que se mantiene con la UODI es más alta que la negativa que se tiene con el cargo de capital, lo que conduce a deducir que el comportamiento del EVA está más ligado a la orientación de la UODI. Esto se puede corroborar cuando se compara el EVA con los índices UODI/ANO y Ko. La UODI es menor que el cargo de capital durante los años en que se destruye valor económico: 2008, 2009, 2011, 2012, 2013 y 2014, y mayor cuando se crea valor: 2010. La UODI sigue una idéntica orientación a la de UAII, y una muy similar a la de la utilidad bruta, solo se diferencia de esta última en 2012, cuando la UODI decae por un aumento considerable de los gastos de venta; algunos otros inductores del UODI se muestran en la sección a de la tabla 12 .

El cargo de capital sigue tendencias similares al Ko y al ANO, en especial con el primero, dado que en 2010 se rompe esta semejanza con el ANO. Aunque el ANO no marca un patrón de comportamiento, sus componentes sí; en particular el AFNO, que tiende a disminuir en este período; mientras que el KTNO cambia de orientación cada dos años. A comienzos del septenio, el KTNO era mayor, pero a finales del mismo fue relevado por el AFNO (parte $b$ de la tabla 12).

El Ko se alinea al comportamiento del Ke, dado que el nivel de endeudamiento no supera el 29,3\%. Además, la relación negativa entre la tasa de impuestos y el Ko es moderada, mientras que este tipo de relación entre el nivel de endeudamiento y el Ko es casi nula. El Ke ha sido superior al costo de la deuda durante el período de estudio y presenta el pico más alto en 2008 (23,1\%) y el más bajo en 2009 (12,2\%); a la vez que el Ki reportó el porcentaje más alto en 2009 (16\%) y el más bajo en el año 2010 (6,6\%); ambos costos no siguen una tendencia por más de tres años (parte $c$ de la tabla 12).

En la tabla 13 se presentan los factores tenidos en cuenta para el cálculo del Ke del sector automotor en Colombia.

\begin{tabular}{lrrrrrrr}
\hline & $\mathbf{2 0 0 8}$ & $\mathbf{2 0 0 9}$ & $\mathbf{2 0 1 0}$ & $\mathbf{2 0 1 1}$ & $\mathbf{2 0 1 2}$ & $\mathbf{2 0 1 3}$ & $\mathbf{2 0 1 4}$ \\
\hline Rf (\%) & 2,2100 & 3,8400 & 3,2900 & 3,2900 & 1,7600 & 3,0400 & 2,1700 \\
$\beta_{\mathrm{L}}$ & 1,3169 & 1,9059 & 2,1784 & 2,3200 & 2,4166 & 1,8660 & 1,7574 \\
Rm (\%) & 7,8900 & 7,6100 & 7,6900 & 7,6900 & 7,5600 & 8,3900 & 8,2400 \\
Ke USA (\%) & 9,7000 & 11,0200 & 12,8800 & 13,5000 & 15,7700 & 13,0200 & 12,8300 \\
RP (\%) & 4,7000 & 2,0000 & 1,3000 & 1,8000 & 1,1200 & 1,6600 & 1,9600 \\
Devaluación (\%) & 7,5700 & $-0,7000$ & 1,5800 & 0,7700 & 1,0000 & 0,4300 & 2,8200 \\
Ke Colombia (\%) & 23,1000 & 12,2000 & 16,0000 & 16,200 & 18,1000 & 15,2000 & 18,0000 \\
\hline
\end{tabular}

Tabla 13

Variables consideradas en la determinación del costo del capital propio Fuente: elaboración propia, con base en EMIS Benchmark (2015), Superintendencia Financiera (2015b) y Damodaran (2015) 
Al descomponer el sistema DuPont, en la relación UODI/ANO, se halla que los índices UODI/Ventas y Ventas/ANO siguen una misma dirección; el primero oscila entre 2,3\% (2013) y 5,1\% (2008), y el segundo fluctúa entre 2,1 veces (2009) y 3,2 veces (2011). El comportamiento de ambos índices y sus dimensiones apuntan a que la rentabilidad de los activos netos operacionales depende tanto de la eficacia en el control de costos y gastos, como de la eficiencia en el uso de los activos netos operacionales ${ }^{6}$ (parte $d$ de la tabla 12).

El VMA del sector automotor colombiano al 1 de enero de 2008 era de $-\$ 6.036$ MM, lo que significa que a pesar de que este sector generó utilidades contables, destruyó valor económico durante el septenio (parte $e$ de la tabla 12).

Al hacer una revisión anual, se comprueba que en todos los años (excepto en 2010) se presentó un porcentaje de pérdida residual, como se muestra en la tabla 14, debido a que en estos años la rentabilidad del activo neto operacional es inferior al costo de capital medio ponderado; lo que explica por qué se destruyó valor económico, a pesar de generar rentabilidades contables positivas.

El EVA/ANO y UODI/EVA presentan valores negativos, a excepción del año 2010, como se aprecia en la tabla 15.

El EVA/ANO muestra que a medida que se avanzaba en el primer trienio se venía destruyendo menos valor por cada peso invertido, hasta llegar a crear $\$ 0,06$ en 2010 , pero en el segundo

6 Esta deducción difiere con la planteada por el análisis del sistema DuPont del ROA, debido a que en la relación UODI/ANO, la rotación de activos es más significativa (tabla 11 y sección d de la tabla 12). trienio la tendencia cambió: se pasó a destruir más valor por peso invertido con el transcurrir de los años; esta tendencia solo se revirtió ligeramente en 2014. La interpretación de relación UODI/EVA tiene mayor sentido cuando es positiva; no obstante, los resultados muestran que de los años en que se destruyó valor, 2013 es cuando menos UODI se necesitó $(\$ 0,98)$ para destruir un peso de EVA; en cambio en 2010, año en que se creó valor, por cada $\$ 3,21$ de UODI se creaba un peso de EVA (tabla 15).

El EVA de capital propio presenta dos tendencias en el septenio: la primera parte destruyendo valor en 2008, pero reduciéndolo hasta llegar a crear valor en 2010, y la segunda se inicia en 2011 desde cuando se mantuvo una destrucción creciente hasta 2014. Tan solo en 2010 el rendimiento del capital invertido por propietarios compensa su costo de oportunidad (tabla 16).

\section{Análisis y contrastes de los resultados}

En este aparte se comparan los principales resultados de esta investigación sobre el sector automotor colombiano (SAC) con los datos de la industria automotriz colombiana (IAC) reportados por la revista Dinero (2009, 2010, 2011, 2012, 2013, 2014 y 2015), los hallazgos del estudio de Jorge Alberto Rivera-Godoy y Ana Milena Padilla-Ospina (2015) en el sector de ensamble (SE), lo encontrado por Jorge Alberto Rivera-Godoy y Ana Milena PadillaOspina (2016) en el sector de autopartes (SA) y la información del sector automotor de Estados Unidos de América (SAEEUU) de la base de datos de Aswath Damodaran (2015), en indicado- 


\begin{tabular}{lrrrrrrr}
\hline & $\mathbf{2 0 0 8}$ & $\mathbf{2 0 0 9}$ & $\mathbf{2 0 1 0}$ & $\mathbf{2 0 1 1}$ & $\mathbf{2 0 1 2}$ & $\mathbf{2 0 1 3}$ & $\mathbf{2 0 1 4}$ \\
\hline UODI/ ANO (\%) & 12,2 & 6,4 & 20,3 & 12,9 & 11,0 & 6,4 & 9,6 \\
Ko (\%) & 19,5 & 10,4 & 14,0 & 14,2 & 15,7 & 13,0 & 15,4 \\
Utilidad o pérdida residual (\%) & $-7,3$ & $-3,9$ & 6,4 & $-1,3$ & $-4,6$ & $-6,6$ & $-5,8$ \\
\hline
\end{tabular}

Tabla 14

Porcentaje de utilidad o pérdida residual

Fuente: elaboración propia, con base en EMIS Benchmark (2015)

\begin{tabular}{lccccccc}
\hline & $\mathbf{2 0 0 8}$ & $\mathbf{2 0 0 9}$ & $\mathbf{2 0 1 0}$ & $\mathbf{2 0 1 1}$ & $\mathbf{2 0 1 2}$ & $\mathbf{2 0 1 3}$ & $\mathbf{2 0 1 4}$ \\
\hline EVA/ANO & $(0,07)$ & $(0,04)$ & 0,06 & $(0,01)$ & $(0,05)$ & $(0,07)$ & $(0,06)$ \\
UODI/EVA & $(1,68)$ & $(1,64)$ & 3,21 & $(9,95)$ & $(2,39)$ & $(0,98)$ & $(1,64)$ \\
\hline
\end{tabular}

Tabla 15

Otros indicadores de control del EVA

Fuente: elaboración propia, con base en EMIS Benchmark (2015)

\begin{tabular}{lccrcccc}
\hline & $\mathbf{2 0 0 8}$ & $\mathbf{2 0 0 9}$ & $\mathbf{2 0 1 0}$ & $\mathbf{2 0 1 1}$ & $\mathbf{2 0 1 2}$ & $\mathbf{2 0 1 3}$ & $\mathbf{2 0 1 4}$ \\
\hline EVA del capital propio (MM\$) & $(3.405,0)$ & $(3.227,0)$ & 211,0 & $(2.533,0)$ & $(3.364,0)$ & $(4.162,0)$ & $(5.661,0)$ \\
ROE $(\%)$ & 13,7 & 2,9 & 16,6 & 9,6 & 10,6 & 5,9 & 6,1 \\
Ke $(\%)$ & 23,1 & 12,2 & 16,0 & 16,2 & 18,1 & 15,2 & 18,0 \\
\hline
\end{tabular}

Tabla 16

EVA promedio por empresa, generado por el capital propio

Fuente: elaboración propia, con base en EMIS Benchmark (2015) y Aswath Damodaran (2015)

$\mathrm{MM} \$=$ cifras en millones de pesos colombianos

res financieros relacionados con el crecimiento, eficiencia, eficacia, efectividad y el valor económico agregado.

\subsection{Crecimiento}

El crecimiento detectado en este estudio de las ventas y los activos del SAC desde 2010 hasta 2014, con una caída en 2013, coincide con los datos de la IAC, y los hallazgos del SA, y difiere un poco de lo encontrado en el SE, pues estos se reducen entre 2012-2013. Además, la fluctuación de utilidades netas del SAC es similar a lo que se muestra en la IAC, el SE y el SA; en este último, la fluctuación es más moderada (tabla 17).

\subsection{Eficiencia}

La variabilidad y magnitud de la rotación de activos totales del SAC es similar al reportado en la IAC; semejante en las fluctuaciones, pero con índices más bajos al compararse con el SE; y con rotaciones más altas que el SA, que mantiene este indicador casi invariable alrededor del 1,1. Esto demuestra que el sector automotor colombiano mantiene una relativa estabilidad en la intensidad con que usa sus activos para generar ventas; además, esta eficiencia está por encima del sector de autopartes, pero por debajo del sector ensamble (tabla 18). 


\begin{tabular}{|c|c|c|c|c|c|c|c|}
\hline Indicador & 2008 & 2009 & 2010 & 2011 & 2012 & 2013 & 2014 \\
\hline \multicolumn{8}{|c|}{ SAC } \\
\hline Ventas (MM\$) & 105.189 & 82.973 & 105.910 & 129.940 & 138.582 & 131.158 & 134.813 \\
\hline Activos (MM\$) & 69.640 & 65.541 & 66.527 & 78.035 & 86.162 & 85.955 & 92.482 \\
\hline Utilidad neta (MM\$) & 4.954 & 1.018 & 5.936 & 3.633 & 4.385 & 2.623 & 2.827 \\
\hline \multicolumn{8}{|c|}{ IAC } \\
\hline Ventas (MM\$) & 169.858 & 142.100 & 177.654 & 194.254 & 234.370 & 213.974 & 229.216 \\
\hline Activos (MM\$) & 114.455 & 116.001 & 118.435 & 118.666 & 143.825 & 131.228 & 148.249 \\
\hline Utilidad neta (MM\$) & 7.834 & 2.552 & 9.957 & 5.314 & 7.404 & 4.322 & 7.301 \\
\hline \multicolumn{8}{|c|}{ SE } \\
\hline Ventas (MM\$) & 894.417 & 540.422 & 679.968 & 821.836 & 763.594 & 750.701 & n.d. \\
\hline Activos (MM\$) & 503.706 & 362.558 & 348.322 & 396.030 & 389.325 & 388.727 & n.d. \\
\hline Utilidad neta (MM\$) & 38.199 & $(832)$ & 34.919 & 11.014 & 19.768 & 8.264 & n.d. \\
\hline \multicolumn{8}{|c|}{ SA } \\
\hline Ventas (MM\$) & 30.532 & 29.936 & 34.153 & 38.365 & 40.924 & 37.288 & 42.037 \\
\hline Activos (MM\$) & 28.580 & 31.157 & 31.303 & 35.947 & 37.906 & 40.080 & 44.444 \\
\hline Utilidad neta (MM\$) & 1.809 & 1.233 & 2.313 & 2.656 & 1.982 & 1.768 & 2.195 \\
\hline
\end{tabular}

Tabla 17

Comparación de los indicadores de crecimiento promedio del sector

Fuente: tomado de las tablas 2, 4, 6 y 8

MM\$ = cifras en millones de pesos colombianos

n.d. = no hay datos

\begin{tabular}{|c|c|c|c|c|c|c|c|}
\hline Indicador & 2008 & 2009 & 2010 & 2011 & 2012 & 2013 & 2014 \\
\hline \multicolumn{8}{|c|}{ SAC } \\
\hline Rotación de activos totales (veces) & 1,5 & 1,2 & 1,6 & 1,8 & 1,7 & 1,5 & 1,5 \\
\hline \multicolumn{8}{|c|}{$\mathrm{IAC}$} \\
\hline Rotación de activos totales (veces) & 1,5 & 1,2 & 1,5 & 1,6 & 1,6 & 1,6 & 1,5 \\
\hline \multicolumn{8}{|c|}{ SE } \\
\hline Rotación de activos totales (veces) & 1,8 & 1,2 & 1,9 & 2,2 & 1,9 & 1,9 & n.d. \\
\hline \multicolumn{8}{|c|}{ SA } \\
\hline Rotación de activos totales (veces) & 1,1 & 1,0 & 1,1 & 1,1 & 1,1 & 1,0 & 1,0 \\
\hline
\end{tabular}

Tabla 18

Comparación de los indicadores de eficiencia del sector

Fuente: tomado de las tablas 2, 4, 6 y 9

n.d. = no hay datos 


\subsection{Eficacia}

Los márgenes de utilidad neta del SAC hallados en el septenio son parecidos a los de la IAC, caracterizados por los altibajos presentados cada año. Estos porcentajes del SAC también oscilan de manera semejante a los del SE, aunque con valores mayores; mientras que al hacer la comparación entre el SAC y el SA, se observa que el primero tuvo porcentajes menores, y las orientaciones de sus variaciones, aunque semejantes, difieren en los años 2011 y 2012. Con esto se puede deducir que dada la variabilidad de los márgenes de utilidad neta, el sector automotor en Colombia no logra mantener un patrón de comportamiento con el que se le pueda hacer un seguimiento a la eficacia de su gestión en el control de costos y gastos; lo mismo sucede con los otros sectores afines como el de ensamble y autopartes; sin embargo, en este período de estudio la eficacia del sector automotor es mayor que la del sector ensamble y menor que la del sector autopartes (tabla 19).

\begin{tabular}{|c|c|c|c|c|c|c|c|}
\hline Indicador & 2008 & 2009 & 2010 & 2011 & 2012 & 2013 & 2014 \\
\hline \multicolumn{8}{|c|}{ SAC } \\
\hline Margen de utilidad neta (\%) & 4,7 & 1,2 & 5,6 & 2,8 & 3,2 & 2,0 & 2,1 \\
\hline \multicolumn{8}{|c|}{ IAC } \\
\hline Margen de utilidad neta (\%) & 4,6 & 1,8 & 5,6 & 2,7 & 3,2 & 2,0 & 3,2 \\
\hline \multicolumn{8}{|c|}{ SE } \\
\hline Margen de utilidad neta (\%) & 4,3 & $-0,2$ & 5,1 & 1,3 & 2,6 & 1,1 & n.d. \\
\hline \multicolumn{8}{|c|}{ SA } \\
\hline Margen de utilidad neta (\%) & 5,9 & 4,1 & 6,8 & 6,9 & 4,8 & 4,7 & 5,2 \\
\hline
\end{tabular}

Tabla 19

Comparación de los indicadores de eficacia del sector Fuente: tomado de las tablas 2, 4, 6 y 10 n.d. = no hay datos

\subsection{Apalancamiento financiero}

El apalancamiento financiero del SAC sigue un comportamiento similar al de la IAC en el septenio: en los tres primeros años se reduce, en los dos siguientes aumenta, y en los dos últimos varía. Este comportamiento es semejante al presentado en el SE (a excepción de 2009) y en el SA (exceptuando 2012). En todos los años se observa que el apalancamiento financiero del sector automotor en Colombia es superior al del sector autopartes, pero inferior al del sector ensamble (tabla 20).

\subsection{Efectividad}

El ROE del SAC es similar al de la IAC, un poco más en su comportamiento de cambio de orientación anual, que en sus porcentajes? Pero cuando esta comparación se hace con el SAEEUU, se observa que en este último se reduce en el primer trienio, y luego fluctúa, pero, además, sus variaciones son más profundas.

7 Las diferencias menores del ROE obedecieron principalmente al menor margen de utilidad neta del SAC, cuando su ROE fue inferior al del IAC (2009 y 2014); y al mayor apalancamiento financiero del SAC, cuando su ROE fue superior al del IAC (2010-2012). 
Al comparar el ROE del SAC con el del SE, se halla que estos índices concuerdan en sus oscilaciones anuales, aunque los porcentajes del SE son más altos en los años 2008, 2010 y 2011. De otro lado, cuando la comparación del ROE se hace entre el SAC y el SA, la concordancia entre sus orientaciones anuales se mantiene, menos en 2012, con porcentajes más altos en el SA en los años 2009, 2011 y 2013 (tabla 21).

\begin{tabular}{lccccccc}
\hline Indicador & $\mathbf{2 0 0 8}$ & $\mathbf{2 0 0 9}$ & $\mathbf{2 0 1 0}$ & $\mathbf{2 0 1 1}$ & $\mathbf{2 0 1 2}$ & $\mathbf{2 0 1 3}$ & $\mathbf{2 0 1 4}$ \\
\hline \multicolumn{7}{c}{ SAC } \\
\hline Ap.F (\%) & 192,4 & 190,9 & 184,3 & 191,4 & 197,6 & 192,9 & 193,3 \\
\hline \multicolumn{7}{c}{ IAC } \\
\hline Ap.F (\%) & 202,4 & 186,2 & 178,1 & 187,7 & 189,9 & 181,4 & 183,2 \\
\hline \multicolumn{7}{c}{ SE } \\
\hline Ap.F (\%) & 202,7 & 206,2 & 200 & 211,8 & 226,9 & 218,4 & n.d. \\
\hline \multicolumn{7}{c}{ SA } \\
\hline Ap.F (\%) & 178,5 & 171,9 & 167,2 & 168,2 & 164,1 & 162,0 & 165,4 \\
\hline
\end{tabular}

Tabla 20

Comparación de los indicadores de apalancamiento financiero (Ap.F) del sector Fuente: tomado de las tablas 2, 4, 6 y 11 n.d. = no hay datos

\begin{tabular}{lccccccc}
\hline Indicador & $\mathbf{2 0 0 8}$ & $\mathbf{2 0 0 9}$ & $\mathbf{2 0 1 0}$ & $\mathbf{2 0 1 1}$ & $\mathbf{2 0 1 2}$ & $\mathbf{2 0 1 3}$ & $\mathbf{2 0 1 4}$ \\
\hline \multicolumn{7}{c}{ SAC } \\
\hline ROE (\%) & 13,7 & 2,9 & 16,6 & 9,6 & 10,6 & 5,9 & 6,1 \\
\hline \multicolumn{7}{c}{ IAC } \\
\hline ROE (\%) & 13,9 & 4,1 & 15,0 & 8,4 & 9,8 & 6,0 & 9,0 \\
\hline \multicolumn{7}{c}{ SE } \\
\hline ROE (\%) & 15,4 & $-0,4$ & 19,6 & 6,3 & 11,4 & 4,6 & n.d. \\
\hline \multicolumn{7}{c}{ SA } \\
\hline ROE (\%) & 11,3 & 7,1 & 12,3 & 13,2 & 8,8 & 7,3 & 8,6 \\
\hline \multicolumn{7}{c}{ SAEEUU } \\
\hline ROE (\%) & 14,0 & $-13,3$ & $-18,8$ & 11,7 & 11,2 \\
\hline
\end{tabular}

Tabla 21

Comparación de los indicadores de efectividad del sector

Fuente: tomado de las tablas 2, 4, 6, 7 y 11

n.d. = no hay datos

Estos resultados permiten deducir que el SAC mantiene un nivel de efectividad más moderado en comparación con el SAEEUU, pues mientras el primero fluctúa entre 2,9\% y $16 \%$, el segundo oscila entre $-18,8 \%$ y $17,6 \%$. 
En el contexto nacional, el SAC es superado en su efectividad de manera alterna en cada uno de los años del septenio, bien sea por el SE o por el SA. Los factores que influyen para esta superación son la rotación de activos y el apalancamiento financiero en el caso del SE, y el margen de utilidad neta en el caso del SA.

\subsection{Valor económico agregado}

El comportamiento del EVA total del SAC es diferente al del SAEEUU: en Colombia se destruye valor en seis años, mientras que en Estados Unidos de América se destruye valor en tres $a_{n}{ }^{8}$; lo mismo sucede cuando se compara el EVA del capital (tabla 22: secciones $a, b, c$ y $d$ ).

$\mathrm{Al}$ revisar los inductores del EVA total, el Ko es mayor en Colombia en todos los años, pero la relación UODI/ANO solo es superior en cinco años; precisamente en los dos años (2011 y 2013) cuando esta relación es superior en SAEEUU, se crea valor en Estados Unidos y se destruye en Colombia. En los otros dos años creadores de valor del SAEEUU (2008 y 2014) y destructores del SAC, el Ko del SAEEUU es muy inferior al SAC.

$\mathrm{Al}$ analizar el Ke y el ROE en el EVA de capital del sector automotor en ambos países, el Ke es superior en el SAC en todos los años, pero su ROE es inferior cuando este valor es positivo. El SAC no registra ROE negativos durante el septenio, como sucede en el SAEEUU en los años 2009 y 2010.

Al comparar el EVA total del SAC con el del SE, sus tendencias son similares: con una mejora en los tres primeros años, y aumento de

8 El VMA del septenio para el SAC fue negativo, mientras que para el SAEEUU fue positivo. destrucción de valor en los siguientes años. Esta tendencia es algo parecida cuando se compara el EVA del capital (secciones $a, c, e$ y $f$ de la tabla 22).

$\mathrm{Al}$ examinar el EVA total, el Ko es mayor en el SAC, pero su índice UODI/ANO es menor que el del SE (excepto en 2009); simultáneamente, cuando se analiza el EVA del capital, el Ke es mayor en el SAC, pero el ROE solo es menor en tres años: 2008, 2010 y 2012.

Cuando se coteja el EVA total del SAC con el del SA, siguen tendencias algo semejantes, con orientaciones diferentes en los años 2010 y 2014. Lo mismo ocurre con el EVA del capital, pero en este caso la diferencia se presenta en los años 2011 y 2013 (secciones $a, c, g$ y $h$ de la tabla 22).

Al confrontar el Ko y la relación UODI/ ANO, ambos son más altos en el SAC, menores en 2008 para el primero, y en 2009 para la segunda; entretanto, cuando se contraponen el Ke y el ROE, se detecta que son más altos en el SAC solo en los años 2008, 2010 y 2012 el primero, y de 2011 a 2014, el segundo.

\section{Conclusiones}

Del análisis realizado del sector automotor colombiano para el período 2008-2014, se encontró un crecimiento de las ventas y de los activos a partir de 2010, con una reducción en 2013, haciendo frente a los nuevos retos que ocasionaron las firmas de los tratados de libre comercio, la crisis andina y la crisis mundial (Cantor, Herrera \& Sánchez, 2012, p. 12; Castro-Patiño, 2009; Restrepo-Cardona, 2010). El comportamiento de la efectividad de lograr utilidades 


\begin{tabular}{|c|c|c|c|c|c|c|c|}
\hline & 2008 & 2009 & 2010 & 2011 & 2012 & 2013 & 2014 \\
\hline \multicolumn{8}{|c|}{ EVA: SAC } \\
\hline EVA $(\mathrm{MM} \$)$ & $(3.187,0)$ & $(1.522,0)$ & $2.410,0$ & $(524,0)$ & $(2.166,0)$ & $(3.061,0)$ & $(2.745,0)$ \\
\hline UODI/ ANO (\%) & 12,2 & 6,4 & 20,3 & 12,9 & 11,0 & 6,4 & 9,6 \\
\hline Кo (\%) & 19,5 & 10,4 & 14,0 & 14,2 & 15,7 & 13,0 & 15,4 \\
\hline ANO (MM\$) & $43.891,0$ & $38.640,0$ & $37.896,0$ & $40.452,0$ & $46.755,0$ & $46.696,0$ & $46.940,0$ \\
\hline \multicolumn{8}{|c|}{ EVA: SAEEUU } \\
\hline EVA (MMUS\$) & $28.104,00$ & $(42.032,00)$ & $(36.984,00)$ & $56.676,00$ & $(3.375,00)$ & $6.508,00$ & 722,00 \\
\hline UODI/ANO (\%) & 10,80 & 1,90 & 0,40 & 15,30 & 7,00 & 12,00 & 5,90 \\
\hline Ko $(\%)$ & 6,21 & 8,71 & 7,39 & 6,91 & 7,49 & 8,41 & 5,63 \\
\hline ANO (MMUS\$) & $616.692,00$ & $619.340,00$ & $531.646,00$ & $675.110,00$ & $703.684,00$ & $179.137,00$ & $276.728,00$ \\
\hline \multicolumn{8}{|c|}{ EVA del capital: SAC } \\
\hline EVA de capital propio (MM\$) & $(3.405,0)$ & $(3.227,0)$ & 211,0 & $(2.533,0)$ & $(3.364,0)$ & $(4.162,0)$ & $(5.661,0)$ \\
\hline $\mathrm{ROE}(\%)$ & 13,7 & 2,9 & 16,6 & 9,6 & 10,6 & 5,9 & 6,1 \\
\hline $\operatorname{Ke}(\%)$ & 23,1 & 12,2 & 16,0 & 16,2 & 18,1 & 15,2 & 18,0 \\
\hline Patrimonio (MM\$) & $36.189,0$ & $34.615,0$ & $37.028,0$ & $38.499,0$ & $44.590,0$ & $44.646,0$ & $47.669,0$ \\
\hline \multicolumn{8}{|c|}{ EVA de capital propio: SAEEUU } \\
\hline $\begin{array}{l}\text { EVA de capital propio } \\
(\text { MMUS\$) }\end{array}$ & $6.488,0$ & $(40.526,0)$ & $(58.539,0)$ & 436,0 & $(2.263,0)$ & $9.428,0$ & $7.329,0$ \\
\hline ROE (\%) & 14,0 & $(13,3)$ & $(18,8)$ & 11,7 & 11,2 & 20,2 & 17,6 \\
\hline $\operatorname{Ke}(\%)$ & 11,3 & 11,4 & 10,8 & 11,6 & 11,8 & 9,8 & 9,0 \\
\hline Patrimonio (MMUS\$) & $239.879,0$ & $164.353,0$ & $197.550,0$ & $360.311,0$ & $396.773,0$ & $90.158,0$ & $85.464,0$ \\
\hline \multicolumn{8}{|c|}{ EVA: SE } \\
\hline EVA $(\mathrm{MM} \$)$ & $(13.571,00)$ & $(5.953,00)$ & $34.262,00$ & $10.736,00$ & $3.806,00$ & $(3.490,00)$ & n.d. \\
\hline UODI/ ANO (\%) & 14,21 & 6,07 & 31,31 & 18,00 & 15,51 & 9,48 & n.d. \\
\hline Ko $(\%)$ & 18,80 & 9,20 & 11,80 & 11,90 & 13,40 & 11,40 & n.d. \\
\hline ANO $(\mathrm{MM} \$)$ & $298.006,00$ & $190.344,00$ & $175.484,00$ & $176.085,00$ & $184.470,00$ & $181.049,00$ & n.d. \\
\hline \multicolumn{8}{|c|}{ EVA de capital propio: SE } \\
\hline EVA $(\mathrm{MM} \$)$ & $(3.892,0)$ & $(4.973,0)$ & $3.224,0$ & $(2.815,0)$ & $(1.366,0)$ & $(3.357,0)$ & n.d. \\
\hline ROE (\%) & 15,4 & $(0,4)$ & 19,6 & 6,3 & 11,4 & 4,6 & n.d. \\
\hline $\operatorname{Ke}(\%)$ & 21,8 & 10,5 & 12,6 & 13,0 & 14,7 & 12,7 & n.d. \\
\hline Patrimonio (MM\$) & $248.536,0$ & $171.567,0$ & $183.922,0$ & $167.596,0$ & $178.592,0$ & $177.723,0$ & n.d. \\
\hline \multicolumn{8}{|c|}{ EVA: SA } \\
\hline EVA (MM\$) & $(2.057,0)$ & $(731,0)$ & $(994,0)$ & $(1.239,0)$ & $(2.018,0)$ & $(2.280,0)$ & $(2.561,0)$ \\
\hline UODI/ ANO (\%) & 9,4 & 6,9 & 8,8 & 7,6 & 6,0 & 3,2 & 5,1 \\
\hline Кo (\%) & 19,8 & 10,3 & 13,6 & 13,1 & 14,0 & 11,9 & 14,4 \\
\hline ANO $(\mathrm{MM} \$)$ & $19.853,0$ & $21.103,0$ & $20.698,0$ & $22.501,0$ & $25.237,0$ & $26.340,0$ & $27.656,0$ \\
\hline \multicolumn{8}{|c|}{ EVA de capital propio: SA } \\
\hline EVA de capital propio (MM\$) & $(2.000,0)$ & $(956,0)$ & $(822,0)$ & $(450,0)$ & $(1.938,0)$ & $(1.800,0)$ & $(2.428,0)$ \\
\hline $\mathrm{ROE}(\%)$ & 11,3 & 7,1 & 12,3 & 13,2 & 8,8 & 7,3 & 8,6 \\
\hline $\operatorname{Ke}(\%)$ & 23,8 & 12,2 & 16,8 & 15,4 & 17,0 & 14,7 & 17,7 \\
\hline Patrimonio (MM\$) & $16.007,0$ & $18.737,0$ & $18.615,0$ & $21.352,0$ & $23.652,0$ & $24.483,0$ & $26.621,0$ \\
\hline
\end{tabular}

Tabla 22

Comparación del EVA promedio por empresa del sector y sus inductores

Fuente: tomado de las tablas 3, 5, 7, 12 y 16; Jorge Alberto Rivera-Godoy y Ana Milena Padilla-Ospina (2015, 2016)

$\mathrm{MM} \$=$ cifras en millones de pesos colombianos

MMUS\$ = millones de dólares

n.d. = no hay datos 
sobre el capital depende de tres factores de acuerdo con la siguiente escala de importancia: 1. Eficacia. 2. Apalancamiento financiero y 3. Eficiencia. Estos resultados son grosso modo, concordantes con los de la IAC, el SA y el SE.

La efectividad del SAC es superada de manera alterna en cada uno de los años del septenio por el SE o por el SA, debido a una mayor eficiencia y apalancamiento financiero del SE o a una mayor eficacia del SA.

A pesar de que en el septenio el sector automotor colombiano tuvo utilidades contables en todos los años, solo logró crear EVA en uno de ellos (2010) y dejó como saldo un VMA negativo. La tendencia fue mejorar el EVA en los tres primeros años, pero en los cuatro siguientes se cambió la directriz a una destrucción ascendente del EVA. Este hallazgo contrasta con lo sucedido con este mismo sector en Estados Unidos, donde se creó valor en cuatro años, pero su tendencia es relativamente semejante a lo que se presentó en los sectores de ensamble y autopartes en Colombia. El comportamiento del EVA del sector automotor en Colombia estuvo más relacionado con la orientación del indicador UODI/ANO que con el Ko; y el primero dependió tanto de la eficacia en el control de costos y gastos, como de la eficiencia en el uso de los activos netos operacionales.

En este trabajo de investigación se presentaron los factores que han influido en el desempeño del sector automotor colombiano para una muestra heterogénea de empresas. Sin embargo, este estudio podría complementarse con estudios similares para un grupo de empresas de la misma edad, tamaño, ubicación geográfica o subsector.

\section{Referencias}

Asociación Nacional de Empresarios de Colombia, ANDI, Cámara de la Industria Automotriz de la ANDI (s.f.). Industria automotriz. Disponible en: http://www.andi.com.co/ cinau

BBVA Research, América del Sur, Colombia (2015). Situación automotriz Colombia 2015. Disponible en: https://www.bbvaresearch. com/public-compuesta/situacionautomotriz-colombia-2015/

Cantor, Natalia; Herrera, Juan Pablo \& Sánchez, Dennis (2012). Estudio del sector automotor de Colombia. Estudios de mercado. Documento de trabajo 1, Grupo de Estudios Económicos, Superintendencia de Industria y Comercio, SIC. Disponible en: http://www.sic.gov.co/ recursos_user/documentos/promocion_ competencia/Estudios_Economicos/ Estudios_Economicos/Estudios_Mercado/ Estudiosobreelsectorautomotor.pdf Castro-Patiño, Luisa Fernanda (2009). Industria automotriz, adelante a pesar de la crisis mundial. Revista Metal Actual, 13, 14-19. Disponible en: http://www.metalactual. com/ediciones.php?ed_id=13

Damodaran, Aswath (2015). [Base de datos en línea]. Disponible en: http://pages.stern. nyu.edu/ adamodar/

Diario Portafolio, Redacción Economía y Negocios (23 de mayo de 2014). Mazda ahora acelera con la importación de vehículos. Una nueva firma comercializará la marca japonesa en Colombia. Diario Portafolio. Disponible en: http://www.portafolio.co/negocios/ 
empresas/mazda-acelera-importacionvehiculos-47136

EMIS (2015). EMIS Benchmark [base de datos en línea]. Disponible en: https://www.emis. $\mathrm{com} / \mathrm{es}$ ? sv $=\mathrm{BCK} \& \mathrm{pc}=\mathrm{CO}$

Ministerio de Comercio, Industria y Turismo, MinCIT (2013). GM Colmotores abre la primera planta productora de vehículos en el país. Disponible en: http://www.mincit.gov. co/publicaciones.php?id=7196\&dPrint $=1$

Ministerio de Comercio, Industria y Turismo, MinCIT, Despacho Negociador Internacional (2014). Las 100 preguntas del acuerdo comercial con Corea del Sur. Disponible en: http://www.mincit.gov.co/publicaciones. php?id=3292

Modigliani, Franco \& Miller, Merton (1963). Corporate Income Taxes and the Cost of Capital: A Correction. The American Economic Review, 53 (3), 433443. Disponible en: https://www2. bc.edu/ chemmanu/phdfincorp/ MF891\%20papers/MM1963.pdf

Procolombia (2016). Inversión en el sector automotriz [sic] en Colombia. Disponible en: http://inviertaencolombia.com.co/sectores/ manufacturas/automotriz.html

Proexport Colombia (2011). Industria automotriz, perfil sectorial. Bogotá: Proexport Colombia, Vicepresidencia de Inversión, Inteligencia de Mercados, Inversión. Disponible en: http://www. inviertaencolombia.com.co/images/stories/ Perfil_Sectorial_Industria_Automotriz_ Junio_2011.pdf

Proexport Colombia (2012). Industria automotriz. Disponible en: http://
portugalcolombia.com/media/IndustriaAutomovel-Fevereiro-2012.pdf

Reina, Mauricio; Oviedo, Sandra \& Moreno, Jonathan, Fedesarrollo (2014). Importancia económica del sector automotor en Colombia. Bogotá: Fedesarrollo, Centro de Investigación Económica y Social. Disponible en: http://www.repository. fedesarrollo.org.co/handle/11445/977, http://www.repository.fedesarrollo.org.co/ bitstream/11445/977/1/Repor_Julio_2014_ Reina_y_Oviedo.pdf

Restrepo-Cardona, Manuel Alejandro (2010). Los efectos de la política comercial: el caso del G-3 y la cuota de importación en el mercado automotriz [sic] colombiano. Vniversitas, 121, 233-262. Disponible en: http:// revistas.javeriana.edu.co/index.php/vnijuri/ article/view/14431/11619

Revista Dinero (29 de mayo de 2009). Ranking 5 mil empresas. Revista Dinero, 326, 176-250.

Revista Dinero (28 de mayo de 2010). Ranking 5 mil empresas. Revista Dinero, 350, 208.

Revista Dinero (25 de mayo de 2011). Ranking 5 mil empresas. Revista Dinero, 374, 235.

Revista Dinero (25 de mayo de 2012). Ranking 5 mil empresas. Revista Dinero, 398, 228.

Revista Dinero (14 de junio de 2013). Ranking 5 mil empresas. Revista Dinero, 423, 230.

Revista Dinero (13 de junio de 2014). Ranking 5 mil empresas. Revista Dinero, 447, 236.

Revista Dinero (12 de junio de 2015). Ranking 5 mil empresas. Revista Dinero, 471, 198, 200.

Rivera-Godoy, Jorge Alberto (2004). Introducción a la administración financiera: fundamentos y aplicaciones para crear valor. Cali: Universidad del Valle. 
Rivera-Godoy, Jorge Alberto \& Alarcón-Morales, Diana Stella (2012). El cargo de capital en la evaluación del desempeño financiero de empresas innovadoras de confecciones de Cali. Estudios Gerenciales, 28 (123), 85100. Disponible en: http://www.icesi.edu. co/revistas/index.php/estudios_gerenciales/ article/view/1209/pdf

Rivera-Godoy, Jorge Alberto \& Padilla-Ospina, Ana Milena (2015). Valor agregado en el sector de ensamble de vehículos en Colombia. Lúmina, 16, 226-249. Disponible en: http://revistasum.umanizales. edu.co/ojs/index.php/Lumina/article/ viewFile/1676/1719

Rivera-Godoy, J. \& Padilla-Ospina, A. (2016). Sector autopartes en Colombia: comportamiento financiero durante el período 2010-2014. Entramado, 12 (1), 12-29. Disponible en: http://www. unilibrecali.edu.co/images/revistaentramado/pdf/pdf_articulos/volumen12_1/ Entramado_19003803_EneroJunio_2016_12-29.pdf

Ross, Stephen A.; Westerfield, Randolph W. \& Jordan, Bradford D. (2014). Fundamentos de finanzas corporativas. $10^{a}$ ed., México: McGraw-Hill Interamericana. Disponible en: http://www.ndp.cl/book.pdf

Stewart, Bennet (2000). En busca del valor. Barcelona: Ediciones Gestión 2000.

Superintendencia de Sociedades (2015a). 14 multinacionales con inversiones recientes en el país registran activos superiores a los $\$ 3$ billones. Disponible en: http:// www.supersociedades.gov.co/noticias/ Paginas/2015/14-multinacionales-coninversiones-recientes-en-el-pa\%C3\%ADs-registran-activos-superiores-a-los-\$3billones.aspx

Superintendencia Financiera (2015b). Tasa de interés y desembolsos por modalidad de crédito. Disponible en: https://www. superfinanciera.gov.co/jsp/loader.jsf?ISer vicio $=$ Publicaciones\&lTipo $=$ publicacione s\&lFuncion=loadContenidoPublicacion\& $\mathrm{id}=60955$

Wild, John J.; Subramanyam, K. R. \& Halsey, Robert F. (2007). Análisis de estados financieros. $9^{\mathrm{a}}$ ed., México: McGrawHill Interamericana. Disponible en: https:/centrouniversitariopasodelnorte. com/greenstone/collect/lae/ import/12022015Analisis\%20de $\% 20$ Estados\%20Financieros\%209ed\%20Wild. pdf

- Fecha de recepción: 3 de septiembre de 2015

- Fecha de aceptación: 23 de abril de 2016

- Disponible en línea: 14 de diciembre de 2016

\section{Para citar este artículo}

Padilla-Ospina, Ana Milena \& Rivera-Godoy, Jorge Alberto (2016). Industria automotriz de Colombia: ¿un motor generador de valor económico agregado? Cuadernos de Contabilidad, 17 (44), 317-348. https:// dx.doi.org/10.11144/Javeriana.cc17-44. iacm 


\section{Anexo 1. Muestra de empresas del sector automotor de Colombia}

\begin{tabular}{|c|c|c|c|c|c|c|c|c|}
\hline NIT & Razón social & 08 & 09 & 10 & 11 & 12 & 13 & 14 \\
\hline \multicolumn{9}{|c|}{ Autopartes } \\
\hline 890920782 & Almacenes y Talleres Moto Precisión S. A. & $\mathrm{X}$ & $\mathrm{X}$ & $\mathrm{X}$ & $\mathrm{X}$ & $\mathrm{X}$ & $\mathrm{X}$ & $\mathrm{x}$ \\
\hline 860507803 & Andina Trim S. A. & $\mathrm{x}$ & $\mathrm{x}$ & $\mathrm{X}$ & $\mathrm{x}$ & $\mathrm{x}$ & $\mathrm{X}$ & $\mathrm{x}$ \\
\hline 860043092 & Arneses y Gomas S. A. & $\mathrm{X}$ & $\mathrm{x}$ & $\mathrm{X}$ & $\mathrm{X}$ & $\mathrm{X}$ & $\mathrm{X}$ & $\mathrm{x}$ \\
\hline 860051728 & Asam Ltda. C.I. & $\mathrm{X}$ & $\mathrm{X}$ & $\mathrm{X}$ & $\mathrm{X}$ & $\mathrm{X}$ & $\mathrm{X}$ & $\mathrm{x}$ \\
\hline 860043983 & Asociados Técnicos Ltda. & $\mathrm{X}$ & $\mathrm{x}$ & $\mathrm{X}$ & $\mathrm{X}$ & $\mathrm{X}$ & $\mathrm{X}$ & $\mathrm{x}$ \\
\hline 800022005 & Autobuses Aga de Colombia S. A. & $\mathrm{x}$ & $\mathrm{x}$ & $\mathrm{x}$ & $\mathrm{X}$ & $\mathrm{X}$ & $\mathrm{x}$ & $\mathrm{x}$ \\
\hline 890900162 & Autoindustrial Camel S. A. & $\mathrm{x}$ & $\mathrm{x}$ & $\mathrm{x}$ & $\mathrm{x}$ & $\mathrm{x}$ & $\mathrm{x}$ & $\mathrm{X}$ \\
\hline 800200635 & Automotrices Titán S. A. & $\mathrm{x}$ & $\mathrm{x}$ & $\mathrm{x}$ & $\mathrm{x}$ & $\mathrm{x}$ & $\mathrm{x}$ & $\mathrm{x}$ \\
\hline 890920168 & Big Limitada & $\mathrm{X}$ & $\mathrm{x}$ & $\mathrm{X}$ & $\mathrm{X}$ & $\mathrm{X}$ & $\mathrm{X}$ & $\mathrm{x}$ \\
\hline 890901866 & Bonem S. A. & $\mathrm{x}$ & $\mathrm{x}$ & $\mathrm{x}$ & $\mathrm{x}$ & $\mathrm{x}$ & $\mathrm{x}$ & $\mathrm{x}$ \\
\hline 860029488 & Bundy Colombia S. A. & $\mathrm{x}$ & $\mathrm{x}$ & $\mathrm{x}$ & & & $\mathrm{x}$ & $\mathrm{x}$ \\
\hline 860067705 & Carrocerías Apolo Alcibiades Peña \& Cía. Ltda. & $\mathrm{x}$ & $\mathrm{x}$ & $\mathrm{x}$ & $\mathrm{X}$ & $\mathrm{X}$ & $\mathrm{x}$ & $\mathrm{x}$ \\
\hline 860045671 & Carrocerías Benfor Ltda. & $\mathrm{X}$ & $\mathrm{x}$ & $\mathrm{x}$ & $\mathrm{X}$ & $\mathrm{x}$ & $\mathrm{x}$ & $\mathrm{x}$ \\
\hline 860012400 & Carrocerías El Sol S.A.S. & $\mathrm{X}$ & $\mathrm{x}$ & $\mathrm{X}$ & $\mathrm{X}$ & $\mathrm{X}$ & $\mathrm{x}$ & \\
\hline 800213451 & Castellanos Equipos Técnicos Ltda. Kastek Ltda. & $\mathrm{X}$ & $\mathrm{X}$ & $\mathrm{X}$ & $\mathrm{X}$ & $\mathrm{X}$ & $\mathrm{X}$ & $\mathrm{x}$ \\
\hline 860004655 & Colombiana de Frenos S. A. Cofre & $\mathrm{x}$ & $\mathrm{x}$ & $\mathrm{x}$ & $\mathrm{X}$ & $\mathrm{X}$ & $\mathrm{x}$ & $\mathrm{x}$ \\
\hline 860530855 & Comercializadora Comet S. A. & $\mathrm{x}$ & $\mathrm{x}$ & $\mathrm{x}$ & $\mathrm{X}$ & $\mathrm{X}$ & $\mathrm{X}$ & $\mathrm{x}$ \\
\hline 860516066 & Comercializadora Franig S.A.S. & $\mathrm{X}$ & & & & & & \\
\hline 860053523 & Compañía Colombiana de Servicio Automotriz S. A. & $\mathrm{X}$ & $\mathrm{x}$ & $\mathrm{x}$ & $\mathrm{X}$ & $\mathrm{X}$ & $\mathrm{x}$ & $\mathrm{x}$ \\
\hline 860515518 & Compañía de Partes y Accesorios Ltda. & $\mathrm{X}$ & $\mathrm{x}$ & $\mathrm{x}$ & $\mathrm{X}$ & $\mathrm{X}$ & $\mathrm{X}$ & $\mathrm{x}$ \\
\hline 860515127 & Control Cables S. A. En Liquidación & $\mathrm{x}$ & $\mathrm{x}$ & $\mathrm{X}$ & & & & \\
\hline 800071617 & Cummins de los Andes S. A. & $\mathrm{x}$ & $\mathrm{x}$ & $\mathrm{X}$ & $\mathrm{x}$ & $\mathrm{x}$ & $\mathrm{x}$ & $\mathrm{x}$ \\
\hline 890304758 & Dite S. A. & $\mathrm{x}$ & $\mathrm{X}$ & $\mathrm{x}$ & $\mathrm{X}$ & $\mathrm{X}$ & $\mathrm{X}$ & $\mathrm{X}$ \\
\hline 860001779 & Drant Larabe Ltda. En Liquidación Judicial & $\mathrm{X}$ & & $\mathrm{x}$ & & & & \\
\hline 890304403 & Ema Holdings S. A. & $\mathrm{x}$ & $\mathrm{x}$ & $\mathrm{x}$ & $\mathrm{X}$ & $\mathrm{X}$ & $\mathrm{X}$ & $\mathrm{x}$ \\
\hline 860071576 & Empaquetaduras Car Ltda. & $\mathrm{x}$ & $\mathrm{x}$ & $\mathrm{x}$ & $\mathrm{x}$ & $\mathrm{x}$ & $\mathrm{x}$ & $\mathrm{x}$ \\
\hline 800207682 & Equipos Agroindustriales Palomino Limitada & $\mathrm{X}$ & $\mathrm{x}$ & $\mathrm{X}$ & $\mathrm{x}$ & & $\mathrm{X}$ & $\mathrm{x}$ \\
\hline 890301886 & Fábrica Nacional de Autopartes S. A., Fanalca & $\mathrm{X}$ & $\mathrm{x}$ & $\mathrm{x}$ & $\mathrm{X}$ & $\mathrm{X}$ & $\mathrm{X}$ & $\mathrm{x}$ \\
\hline 800042706 & Fabripartes S. A. En Liquidación Judicial & $\mathrm{x}$ & $\mathrm{x}$ & $\mathrm{X}$ & $\mathrm{X}$ & & & \\
\hline 860040946 & Faral S. A. En Liquidación & $\mathrm{x}$ & & $\mathrm{x}$ & $\mathrm{X}$ & & & \\
\hline 860012340 & $\begin{array}{l}\text { Fraco - Fábrica Colombiana de Repuestos Automotores } \\
\text { S. A. }\end{array}$ & $\mathrm{x}$ & $\mathrm{x}$ & $\mathrm{x}$ & $\mathrm{X}$ & $\mathrm{x}$ & $\mathrm{x}$ & $\mathrm{x}$ \\
\hline 830070281 & Fundiciones y Componentes Automotores S. A., Fundicom & $\mathrm{X}$ & $\mathrm{X}$ & $\mathrm{X}$ & $\mathrm{X}$ & $\mathrm{X}$ & $\mathrm{X}$ & \\
\hline 891409006 & Germán Gaviria S. y Cía. Ltda. Distrimotos & $\mathrm{x}$ & $\mathrm{x}$ & $\mathrm{X}$ & $\mathrm{X}$ & $\mathrm{X}$ & $\mathrm{X}$ & $\mathrm{x}$ \\
\hline 860090052 & Gipar Internacional S. A. & $\mathrm{x}$ & $\mathrm{X}$ & $\mathrm{X}$ & $\mathrm{X}$ & $\mathrm{X}$ & $\mathrm{X}$ & $\mathrm{x}$ \\
\hline
\end{tabular}




\begin{tabular}{|c|c|c|c|c|c|c|c|c|}
\hline NIT & Razón social & 08 & 09 & 10 & 11 & 12 & 13 & 14 \\
\hline 811000289 & Golden Hawk Industries S. A. & $\mathrm{x}$ & $\mathrm{x}$ & $\mathrm{x}$ & $\mathrm{x}$ & $\mathrm{x}$ & $\mathrm{x}$ & $\mathrm{X}$ \\
\hline 800008116 & Hangar Uno de Colombia Ltda. & & & $\mathrm{X}$ & $\mathrm{x}$ & $\mathrm{X}$ & $\mathrm{X}$ & $\mathrm{x}$ \\
\hline 804000248 & Herramientas Suspensiones y Combustibles S. A. & $\mathrm{X}$ & $\mathrm{X}$ & $\mathrm{X}$ & $\mathrm{x}$ & $\mathrm{x}$ & $\mathrm{x}$ & $\mathrm{X}$ \\
\hline 860039586 & I.P.T. Comercializadora Internacional S. A. & $\mathrm{x}$ & $\mathrm{x}$ & $\mathrm{x}$ & $\mathrm{x}$ & $\mathrm{x}$ & $\mathrm{x}$ & $\mathrm{x}$ \\
\hline 860050501 & Inca Fruehauf Inca S.A. & $\mathrm{x}$ & $\mathrm{x}$ & $\mathrm{x}$ & $\mathrm{X}$ & $\mathrm{X}$ & $\mathrm{X}$ & $\mathrm{x}$ \\
\hline 800143430 & Industria Automotriz del Caribe S. A. & $\mathrm{x}$ & & & $\mathrm{x}$ & & & \\
\hline 817000827 & Industria Central Nacional S. A. En Liquidación & $\mathrm{x}$ & & & & & & \\
\hline 890916911 & Industria Colombiana de Motocicletas Yamaha S. A. & $\mathrm{x}$ & $\mathrm{x}$ & $\mathrm{x}$ & $\mathrm{X}$ & $\mathrm{x}$ & $\mathrm{x}$ & $\mathrm{x}$ \\
\hline 860509777 & Industria Interamericana de Filtros Ltda. & $\mathrm{x}$ & & $\mathrm{x}$ & $\mathrm{x}$ & $\mathrm{x}$ & $\mathrm{x}$ & $\mathrm{x}$ \\
\hline 860404848 & Industrias Faaca Colombia S. A. & $\mathrm{x}$ & $\mathrm{X}$ & $\mathrm{X}$ & $\mathrm{X}$ & $\mathrm{X}$ & $\mathrm{X}$ & $\mathrm{X}$ \\
\hline 890200491 & Industrias Partmo S. A. & $\mathrm{x}$ & $\mathrm{x}$ & $\mathrm{x}$ & $\mathrm{x}$ & $\mathrm{x}$ & $\mathrm{x}$ & $\mathrm{X}$ \\
\hline 830099228 & Latin Tecno S. A. & & $\mathrm{x}$ & $\mathrm{x}$ & $\mathrm{X}$ & $\mathrm{x}$ & $\mathrm{x}$ & $\mathrm{X}$ \\
\hline 860509777 & Interfil S.A.S. & $\mathrm{x}$ & & & & & & \\
\hline 817000727 & Mac del Pacífico S.A.S. & $\mathrm{x}$ & $\mathrm{x}$ & $\mathrm{X}$ & $\mathrm{X}$ & $\mathrm{x}$ & $\mathrm{x}$ & \\
\hline 890907177 & Mafricción S. A. & $\mathrm{x}$ & $\mathrm{x}$ & $\mathrm{x}$ & $\mathrm{x}$ & $\mathrm{x}$ & $\mathrm{x}$ & $\mathrm{x}$ \\
\hline 860013933 & Manufacturas Víctor Gaskets de Colombia S. A. & $\mathrm{x}$ & $\mathrm{x}$ & $\mathrm{x}$ & $\mathrm{x}$ & $\mathrm{x}$ & $\mathrm{x}$ & $\mathrm{X}$ \\
\hline 890209585 & Metalizadora del Oriente Ltda. & $\mathrm{x}$ & $\mathrm{x}$ & $\mathrm{x}$ & $\mathrm{x}$ & $\mathrm{x}$ & $\mathrm{x}$ & $\mathrm{X}$ \\
\hline 890316045 & Multipartes Industrial S. A. & $\mathrm{x}$ & $\mathrm{x}$ & $\mathrm{x}$ & $\mathrm{x}$ & $\mathrm{x}$ & $\mathrm{x}$ & $\mathrm{X}$ \\
\hline 890305761 & Multipartes Limitada & $\mathrm{x}$ & $\mathrm{x}$ & $\mathrm{x}$ & $\mathrm{X}$ & $\mathrm{x}$ & $\mathrm{x}$ & $\mathrm{X}$ \\
\hline 860507000 & Prillantas Ltda. & $\mathrm{x}$ & $\mathrm{x}$ & $\mathrm{x}$ & & & & \\
\hline 800207476 & Productos Microcelulares de Colombia S. A. & $\mathrm{x}$ & $\mathrm{x}$ & $\mathrm{x}$ & $\mathrm{x}$ & $\mathrm{x}$ & $\mathrm{x}$ & \\
\hline 860523227 & Renosa S. A. & $\mathrm{X}$ & $\mathrm{X}$ & $\mathrm{x}$ & $\mathrm{x}$ & $\mathrm{x}$ & $\mathrm{x}$ & $\mathrm{X}$ \\
\hline 890900374 & Repuestos Colombianos S. A. & $\mathrm{x}$ & $\mathrm{x}$ & $\mathrm{x}$ & $\mathrm{x}$ & $\mathrm{x}$ & $\mathrm{x}$ & $\mathrm{x}$ \\
\hline 890307671 & Resortes Hércules S. A. & $\mathrm{x}$ & $\mathrm{x}$ & $\mathrm{x}$ & $\mathrm{x}$ & $\mathrm{x}$ & $\mathrm{x}$ & $\mathrm{X}$ \\
\hline 890310237 & Rogelio Villamizar y Cía. S.C.A. & $\mathrm{x}$ & $\mathrm{x}$ & $\mathrm{X}$ & $\mathrm{X}$ & $\mathrm{x}$ & $\mathrm{x}$ & $\mathrm{x}$ \\
\hline 890318329 & Romarco S. A. & $\mathrm{x}$ & $\mathrm{x}$ & $\mathrm{x}$ & $\mathrm{x}$ & $\mathrm{x}$ & $\mathrm{x}$ & $\mathrm{X}$ \\
\hline 800050088 & Sauto S. A. & $\mathrm{x}$ & $\mathrm{x}$ & $\mathrm{x}$ & $\mathrm{x}$ & $\mathrm{x}$ & $\mathrm{x}$ & $\mathrm{X}$ \\
\hline 860052240 & Servicio Didacol Ltda. & $\mathrm{x}$ & $\mathrm{x}$ & $\mathrm{x}$ & $\mathrm{X}$ & $\mathrm{x}$ & $\mathrm{x}$ & $\mathrm{x}$ \\
\hline 890917801 & Servicios de Vehículos Suramericana S. A. & $\mathrm{x}$ & $\mathrm{x}$ & $\mathrm{x}$ & & & & \\
\hline 860026892 & Servicios Industriales Técnicos S. A. & $\mathrm{X}$ & $\mathrm{X}$ & $\mathrm{X}$ & $\mathrm{X}$ & $\mathrm{x}$ & $\mathrm{x}$ & $\mathrm{X}$ \\
\hline 830033457 & Stewart \& Stevenson de las Américas Colombia Ltda. & $\mathrm{x}$ & $\mathrm{x}$ & $\mathrm{x}$ & $\mathrm{x}$ & $\mathrm{x}$ & $\mathrm{x}$ & $\mathrm{x}$ \\
\hline 800053895 & Superficies Colombia S.A.S. & $\mathrm{x}$ & $\mathrm{x}$ & $\mathrm{x}$ & $\mathrm{x}$ & $\mathrm{x}$ & $\mathrm{x}$ & $\mathrm{X}$ \\
\hline 860519235 & Talleres Autorizados S. A. & $\mathrm{x}$ & $\mathrm{x}$ & $\mathrm{x}$ & $\mathrm{x}$ & $\mathrm{x}$ & $\mathrm{x}$ & $\mathrm{x}$ \\
\hline 860011213 & Talleres Díaz Ltda. & $\mathrm{x}$ & $\mathrm{x}$ & $\mathrm{x}$ & $\mathrm{x}$ & $\mathrm{x}$ & $\mathrm{x}$ & $\mathrm{x}$ \\
\hline 830046588 & Total Quality Management S. A. & & $\mathrm{x}$ & & & & & \\
\hline 860042593 & Toyota Talleres de Servicio Autorizado Limitada & $\mathrm{x}$ & $\mathrm{x}$ & $\mathrm{X}$ & $\mathrm{X}$ & $\mathrm{x}$ & $\mathrm{X}$ & $\mathrm{X}$ \\
\hline 800250328 & Transejes Transmisiones Homocinéticas de Colombia S. A. & $\mathrm{x}$ & $\mathrm{x}$ & $\mathrm{x}$ & $\mathrm{X}$ & $\mathrm{x}$ & $\mathrm{x}$ & $\mathrm{x}$ \\
\hline
\end{tabular}




\begin{tabular}{|c|c|c|c|c|c|c|c|c|}
\hline NIT & Razón social & 08 & 09 & 10 & 11 & 12 & 13 & 14 \\
\hline 860028171 & Trimco S. A. & $\mathrm{X}$ & $\mathrm{X}$ & $\mathrm{X}$ & $\mathrm{X}$ & $\mathrm{X}$ & $\mathrm{X}$ & $\mathrm{X}$ \\
\hline 860518253 & Ultra S. A. & $\mathrm{X}$ & $\mathrm{X}$ & $\mathrm{X}$ & $\mathrm{X}$ & $\mathrm{X}$ & $\mathrm{X}$ & $\mathrm{X}$ \\
\hline 890906197 & Umo S. A. & $\mathrm{X}$ & $\mathrm{X}$ & $\mathrm{X}$ & $\mathrm{X}$ & $\mathrm{X}$ & $\mathrm{X}$ & $\mathrm{X}$ \\
\hline 890310054 & Villamizar Angulo y Cía. S.C.A. & $\mathrm{X}$ & $\mathrm{X}$ & $\mathrm{X}$ & $\mathrm{X}$ & $\mathrm{X}$ & $\mathrm{X}$ & $\mathrm{X}$ \\
\hline 830033403 & Yazaki Ciemel F T Z Ltda. & $\mathrm{X}$ & $\mathrm{X}$ & $\mathrm{X}$ & $\mathrm{X}$ & $\mathrm{X}$ & $\mathrm{X}$ & $\mathrm{X}$ \\
\hline 832000402 & Yazaki Ciemel S. A. & $\mathrm{X}$ & $\mathrm{X}$ & $\mathrm{X}$ & $\mathrm{X}$ & $\mathrm{X}$ & $\mathrm{X}$ & $\mathrm{X}$ \\
\hline \multicolumn{9}{|c|}{ Ensamble } \\
\hline 860038515 & Compañía Colombiana Automotriz S. A. & $\mathrm{X}$ & $\mathrm{X}$ & $\mathrm{X}$ & $\mathrm{X}$ & $\mathrm{X}$ & $\mathrm{X}$ & \\
\hline 860002304 & General Motors Colmotores S. A. & $\mathrm{X}$ & $\mathrm{X}$ & $\mathrm{X}$ & $\mathrm{X}$ & $\mathrm{X}$ & $\mathrm{X}$ & $\mathrm{X}$ \\
\hline 860025792 & Sociedad de Fabricación de Automotores S. A., Sofasa & $\mathrm{X}$ & $\mathrm{X}$ & $\mathrm{X}$ & $\mathrm{X}$ & $\mathrm{X}$ & $\mathrm{X}$ & $\mathrm{X}$ \\
\hline 900416386 & Zona Franca Industrial Colmotores S.A.S. & & & & & $\mathrm{X}$ & $\mathrm{X}$ & $\mathrm{X}$ \\
\hline 890900317 & Autotécnica Colombiana S.A. & $\mathrm{X}$ & & $\mathrm{X}$ & $\mathrm{X}$ & $\mathrm{X}$ & $\mathrm{X}$ & \\
\hline 830040343 & Compañía de Autoensamble Ltda. Nissan & $\mathrm{X}$ & $\mathrm{X}$ & $\mathrm{X}$ & $\mathrm{X}$ & $\mathrm{X}$ & $\mathrm{X}$ & $\mathrm{X}$ \\
\hline 900166896 & Hino Motors Manufacturing Colombia S. A. & & $\mathrm{X}$ & $\mathrm{X}$ & $\mathrm{X}$ & $\mathrm{X}$ & $\mathrm{X}$ & $\mathrm{X}$ \\
\hline 891410137 & Suzuki Motor de Colombia & $\mathrm{X}$ & $\mathrm{X}$ & $\mathrm{X}$ & $\mathrm{X}$ & $\mathrm{X}$ & $\mathrm{X}$ & $\mathrm{X}$ \\
\hline 830044266 & Daimler Colombia S. A. & $\mathrm{X}$ & $\mathrm{X}$ & $\mathrm{X}$ & $\mathrm{X}$ & $\mathrm{X}$ & $\mathrm{X}$ & $\mathrm{X}$ \\
\hline 860038196 & Non Plus Ultra S. A. & & $\mathrm{X}$ & $\mathrm{X}$ & $\mathrm{X}$ & $\mathrm{X}$ & $\mathrm{X}$ & \\
\hline
\end{tabular}

Nota: la X indica los años en que la investigación tuvo en cuenta cada empresa (entre 2008 y 2014). 